Apidologie, 1975, $6(2), 95-120$.

\title{
ZUR KASTENSPEZIFISCHEN ERNÄHRUNG DER WEIBLICHEN BIENENLARVE (APIS MELLIFICA L.)
}

\author{
Spécificité de l'alimentation des larves femelles d'abeille \\ (Apis mellifica L.) selon la caste
}

Karl WEISS

Bayerische Landesanstalt für Bienenzucht, Erlangen

\author{
SUMMARY \\ TO THE CASTE SPECIFIC NUTRITION OF FEMAL HONEY BEE LARVAE \\ (Apis mellifica L.)
}

Young worker larvae fed in vitro with worker jelly enriched with invert sugar developed mainly into worker bees but also into an intermediate and a real queen. Most larvae died in the larval stage. Three days old queen larvae receiving worker jelly in the incubator resulted in queens whereas queen larvae of the same age fed with modified worker jelly in worker cells of a queenright host colony yielded worker bees - however sometimes with enlarged ovaries.

From this investigation it can bee concluded that the queen determining principel of queen jelly is present in the worker jelly too - presumably in a less efficient manner.

\section{ZUSAMMENFASSUNG}

Bei der Fütterung junger Arbeitermaden mit invertzuckerangereichertem Arbeiterfuttersaft im Labor entstanden bei relativ hohen Ausfällen neben einer Anzahl Arbeitsbienen eine deutliche Zwischenform und eine Königin. Dreitägige Königinnenlarven entwickelten sich im Brutschrank, mit Arbeiterfuttersaft gefüttert, zu Königinnen, während sie in Arbeiterzellen eines Wirtsvolkes, wahrscheinlich mit Mischfutter aufgezogen, Arbeiterinnen mit nur gelegentlich vergrößerten Eierstöcken ergaben.

Aus den Versuch $\mathrm{n}$ geht hervor, daß das königinnendeterminierende Prinzip des Weiselfuttersaftes auch im Arbeiterinnenfuttersaft - vermutlich in verminderter Wirkung - vorhanden ist. 
Der Kastenpolymorphismus der Honigbiene tritt in den Phänotypen Königin und Arbeiterin in Erscheinung. Beide Formen entwickeln sich aus dem ontogenetisch gleichartigen, befruchteten Ei. Über die Entwicklungsrichtung entscheidet ausschließlich die Larvenernährung. Die Arbeiterinnenlarve wird in den 3 ersten Tagen ihres Lebens von den Ammenbienen mit Drüsensekreten, dem Futtersaft, gefüttert und erhält danach ein “ Mischfutter ) aus Futtersaft, Pollen und Honig. Dic Königinnenmade bekommt dagegen während ihrer ganzen Freßphase nur Drüsensekret.

Das ist der äußere Sachverhalt. Das Problem der nahrungsgesteuerten Kastendetermination ist komplizierter.

\section{I. - GEGENWÄRTIGER WISSENSSTAND UND PROBLEMSTELLUNG}

Da die Arbeiterinnenmade bis zum Futterwechsel zur Königin umstimmbar ist, nahm man früher an, daß eben dieser Futterwechsel - insbesondere der U̇bergang zur gröberen Pollennahrung — für die weibliche Kastendetermination ausschlaggebend sei. Das trifft aber in dieser Vereinfachung nicht zu. Bienenvölker, die man in Flugzelten ohne Pollen nur mit Zuckerfutter ernährt, sind wenigstens anfänglich noch in der Lage, eine geringe Menge Arbeiterbrut zu erzeugen (Himmer 1927, Whitcomb und Wilson 192\%, Haydak 1935, BUCHNER 1953).

Für die Kastenentstehung der Honigbiene ist auch nicht einfach die von der Larve verzehrte Futtermenge - sei es Futtersaft oder Mischfutter - entscheidend. Wenn etwa in einem neu etablierten Schwarm oder im plötzlich entweiselten Bienenvolk die jungen Arbeiterlarven auf ungewöhnlich großen Futtersaftmengen schwimmen (Kuwabara 1947, Gontarski 1953, Weaver 1956), unterscheiden sich die später ausschlüpfenden Tiere deswegen nicht vom reinen Arbeiterinnentyp. Nach Gontarski (1954) erhalten die älteren Arbeitermaden auch normalerweise soviel Mischfutter, daß sich beim Verdeckeln noch Überreste davon in den Zellen befinden. Andererseits gelingt es, durch Wegnahme des Futtersaftüberschusses aus den Weiselzellen um die Zeit der Deckelung reguläre Königinnen aufzuziehen, die kaum größer als Arbeitsbienen sind (Dönhoff 1859, von Rhein 1933, Haydak 1943, Sмith 1959, JAY 1964). Trotzdem scheint die Nahrungsmenge in der Kastendetermination nicht völlig bedeutunglos zu sein (s. Diskussion).

Die Larvenernährung der beiden Bienenkasten ist nicht nur durch den verschiedenen Einsatz der beiden Futterarten - Futtersaft und Mischfutter - und die Futtermenge kastenspezifisch differenziert - auch der Futtersaft selbst ist qualitätsverschieden. Die Ammenbienen geben im wesentlichen 2 verschiedene Futtersaftkomponenten an die jungen Larven ab, eine ( weiße ) und eine “ klare " (von Rhein 1933). Jung-Hoffmann (1966) beobachtete, daß 
Königinnenmaden während der ganzen Fütterungszeit die beiden Komponenten zu etwa gleichen Teilen bekommen, während die jungen Arbeiterlarven mit weniger weißem Sekret zufrieden sein müssen, welches auch häufig nicht so rein weiß ist wie bei den Weisellarven. Über 3 Tage alte Arbeiterlarven erhalten so gut wie kein weißes Sekret mehr. Für die Qualitätsverschiedenheit der Futtersäfte sprechen auch Unterschiede im frühen Larvenwachstum der beiden Kasten (Stabe 1930, Wang 1965); dazu kommen Unterschiede im Atemtoffwechsel (Melampy und Willis 1939, Shuel und Dixon 1959, 1968, Osanai und Reмвоцд 1968), in der Beschaffenheit des Körpergewebes und der Hämolymphe (Dixon und Shuel 1963, Liu und Dixon 1965, Lue und Dixon 1967) und in den Enzymaktivitäten (Tripathi und Dixon 1968, 1969, Czoppelt und Rembond 1970) der Larven in der Zeit vor dem Futterwechsel. Schließlich konnte die stoffliche Verschiedenheit der beiden Futtersäfte auch noch direkt durch chemische Analyse nachgewiesen werden, auch wenn es sich dabei bisher nur um Quantitätsunterschiede verschiedener, in beiden Futtersäften vorhandener Komponenten handelte (Zusf. Lit. bei Johansson und Johansson 1958, Shuel und Dixon 1960, Towsend und Shuel 1962, Rembold 1964, HAYDAK 1968).

So bieten sich gegenwärtig als ernstzunehmende Determinationsursachen in der Hauptsache zwei Möglichkeiten an. Die eine, welche schon von RHEıN (1933) erwog, nimmt einen bestimmten determinierenden Stoff im Weiselfuttersaft an. Weaver wies 1962 auf das Vorhandensein einer Fraktion im Königinnenfuttersaft hin, welche bei künstlichen Aufzuchtversuchen königinnenbestimmende Wirkung entfalte. Rembold und Hanser (1964) fanden den aktiven Faktor im wasserlöslichen Dialysat des Königinnenfuttersaftes. Vermutlich dieselbe Wirkungskomponente konnte Rembold $(1969,1973)$ auch aus einer Aufbereitung von Seidenspinnerschmetterlingen und verschiedenen anderen Insekten gewinnen. Er vergleicht den Determinationsstoff mit einem adenotropen Hormon, das die kastenbildenden innersekretorischen Vorgänge stimuliert und reguliert. Dabei soll der Stoff in noch geringeren Mengen wirken, als gemeinhin von den Insektenhormonen bekannt ist. Auch wenn dieser “ Determinator " nach Rembold et al. (1974) nicht identisch ist mit dem Juvenilhormon, dessen Bedeutung für die weibliche Kastendetermination der Honigbiene erst kürzlich durch WIrTz und BeEssma (1972) und Wirtz (1973) nachgewiesen wurde, so könnte er doch wie dieses Hormon in der Larve selbst gebildet werden und erst durch das Häutungsgeschehen spurenweise in den Futtersaft gelangen. Er müßte nicht unbedingt von den Ammenbienen stammen, wie man gemeinhin annimmt.

Das würde bedeuten, daß die zweite ernstzunehmende Hypothese der Kastenentstehung diskutabel bleibt, welche HAYDAK (1943) erstmals vertreten hat : Danach wäre nicht ein bestimmter Stoff, sondern das Mengenverhältnis oder vielleicht auch das Minimalvorkommen bestimmter essen- 
tieller Stoffe im Königinnenfuttersaft für die Ausbildung der weiblichen Bienenkasten entscheidend. Beispielsweise fanden Butenandt und Rembold (1957) im Königinnenfuttersaft etwas mehr Lipoide und Shuel und Dixon (1959) mehr Zucker, aber weniger titrierbare Säuren und einen etwas geringeren Proteingehalt als im Arbeiterfuttersaft. Im Weiselfuttersaft besonders reich vertreten sind die Panthotensäure (HAYdaK und Vivino 1950, Lingens und Rembold 1959) und die Pteridinabkömmlinge Biopterin (Butenandt und Rembold 1958, Hanser und Rembold 1960) und Neopterin (Rembold und Buschmanv 1963), welche für sich allein jedoch nicht königinnendeterminierend wirken. Auch der Wassergehalt der beiden Futtersäfte weist Verschiedenheiten auf : er nimmt bei der Königinnenmade mit dem Alter zu, bei der Arbeiterinnenmade dagegen ab (Lit. bei Dietz und HaydaK 1971).

Unter dem Eindruck dieser Stoffverschiebungen glauben Dixon und Shuel (1963) eine bestimmte “ balance of nutrients ) für die Ausbildung der beiden weiblichen Bienenkasten verantwortlich machen zu können. Sie konnten zeigen, daß junge Arbeiterinnenlarven bei deren Aufzucht im Labor mit Futtersaft, der durch Zugabe von Zucker oder (und) aus dem Königinnenfuttersaft extrahierten wasserlöslichen Säuren letzterem ähnlich gemacht wurde, in ihrer larvalen Entwicklung - Wachstum, Atmung, Gewebechemie, Histologie der innersekretorischen Drüsen und der Ovarentwicklung - königinnenhafte Züge aufweisen (Shuel und Dixon 1959, Dixon 1959, Dixon und Shuel 1963, Wang und Shuel 1965, O'Brien und Shuel 1972). Andererseits hat Weaver (1974b) erst kürzlich nachgewiesen, daß auch beträchtliche Änderungen im Gleichgewicht der wichtigsten Nahrungskomponenten des Königinnenfuttersaftes die Entwicklung der Larven zu adulten königlichen Tieren kaum zu gefährden vermag.

Wenn man in Betracht zieht, daß schon in den ersten Lebenstagen der Königinnen - und Arbeiterinnenlarve physiologische Entwicklungsunterschiede nachweisbar sind, muß es Wunder nehmen, daß die entstehenden Imagines in der Ausprägung ihrer Merkmale und ihres Verhaltens so gut wie nicht durch diese frühen Unterschiede der Larvenentwicklung beein flußt werden. Wie eingangs erwähnt wurde, sind Arbeitermaden bis zu einem Alter von etwa 3 1/2 Tagen bei nachfolgender königlicher Ernährung in Königinnen und königinnenähnliche Tiere umstimmbar. Noch aus 2 Tage alten Larven entstehen - mit Ausnahme einer minimalen Verringerung des Körpergewichtes - in allen spezifischen Organen vollkommene Weibchen (WeIss 1971, 1974). Es paßt hierzu, daß die histologische Untersuchung der Königinnen - und Arbeiterinnenlarven bis etwa zur Mitte des dritten Lebenstages keine Unterschiede in der Ausbildung der innersekretorischen Drüsen erkennen läßt (Canetti, Shuel und Dixon 1964, Wang und Shuel 1965, O'Brien und Shuel 1972), und daß auch andere Organe, wie die Ovarien, solange 
völlig indifferent bleiben (Haydak 1943, Wang und Shuel 1965, Shuel und Dixon 1960, Mickey und Melampy 1941).

Die verhältnismäßig lang und $100 \%$ ig mögliche Umstimmbarkeit der Arbeiterinnenlarve zur Königin läßt vermuten, daß die beiden Futtersäfte bezüglich ihrer kastendeterminierenden Eigenschaft sehr ähnlich sind. Danach könnte das königinnenbestimmende Prinzip in beiden Futtersäften, also auch in dem der jungen Arbeiterinnenlarven, enthalten sein.

Um das zu prüfen, gilt es, junge Arbeiterinnenlarven nur mit Arbeiterfuttersaft zu adulten Tieren aufzuziehen. Das ist aber nur in vitro, d.h. mit Hilfe manueller Fütterung im Brutschrank möglich. Zum ersten Mal hat es von Rhein (1933) versucht. Er brachte die Larven aber nicht zur Verpuppung. Sмгтн (1959) machte die gleiche enttäuschende Erfahrung. Heute wissen wir, daß die Ursache für diese Mißerfolge im geringen Zuckergehalt des Arbeiterfuttersaftes begründet lag. Die Arbeiterinnenlarve baut in den ersten Tagen ihrer Entwicklung bei größerem Wassergehalt weniger Reservestoffe in Form von Glykogen und Fetten auf als die Königinnenmade (STraus 1911, Melampy et al. 1940, Dixon und Shuel 1963, Wirtz 1973). Man könnte sie im Vergleich zur Königinnenmade für unterernährt halten (HAYDAK 1943). Erst während der Fütterung mit der stärker zuckerhaltigen Mischnahrung legt sie größere Glykogenvorräte an (Czoppest und Rembold 1967), wodurch die Puppenentwicklung gewährleistet erscheint. Wie Shuel und Dixon (1968) feststellten, enthält der Königinnenfuttersaft $12 \%$, der Arbeiterinnenfuttersaft dagegen nur $4 \%$ Zucker. Diesen beiden Autoren ist es auch gelungen, Arbeiterinnenmaden durch ausschließliche Verfütterung von Arbeiterinnenfuttersaft mit Zuckerzusatz zur Verpuppung zu bringen. Allerdings entwickelten sich auf diese Weise bedeutend weniger Puppen (von 41 Larven nur 8) als bei den vergleichsweise mit Königinnenfuttersaft gefütterten Larven (41: 15). Auch wird über die Kastenzugehörigkeit der Puppen nichts berichtet. Wahrscheinlich waren es Arbeiterpuppen, da die Maden bei Versuchsbeginn mit 3 1/2 Tagen schon vordeterminiert gewesen sein konnten. - Schon vor Shuel und Dixon wollen Rembold und Hanser (1964) Arbeiterlarven mit Arbeiterfuttersaft im Brutschrank aufgezogen und ausschließlich Arbeitsbienen erhalten haben. Wie ihnen die Aufzucht gelang, ist nicht ganz klar, da sie von einer Zuckerbeigabe zum Futtersaft nichts erwähnen. Möglicherweise hat es sich bei dem - nach ihren Angaben aus Zellen 3-tägiger Arbeiterinnenmaden gewonnenen - “ Futtersaft 》 bereits um Mischfutter gehandelt.

Die vorhandene Literatur über die Aufzucht von Imagines mit Arbeiterinnenfuttersaft ist nicht befriedigend, besonders weil über die Kastenausbildung der Aufzuchtprodukte nichts Sicheres bekannt ist. Diesem Mangel versuchte ich in der vorliegenden Arbeit abzuhelfen, um damit gleichzeitig 
etwas über die kastenbestimmende Wirkung des Arbeiterfuttersaftes zu erfahren.

\section{II. - METHODE}

\section{a) Gewinnung des Futtersaftes}

Voraussetzung für die geplanten Aufzuchtversuche in vitro war die Gewinnung von genügend altersdatiertem kastenspezifischem Arbeiterinnenfuttersaft - der zudem in möglichst frischem Zustand verfüttert werden sollte.

Der Ablauf der Futtersaftgewinnung war streng geordnet. Jeden Mittwoch um 8.00 Uhr sperrte ich in mehreren Völkern gut legende Königinnen mit Hilfe von Aufsteckrahmen aus Absperrgitter auf helle Waben ab, am Donnerstag zur selben Zeit überführte ich sie auf neue Waben, und von Freitag bis Samstag hatten sie Zeit, auf einem dritten Wabensatz zu stiften. Am Samstag um 8.00 Uhr wurden die Königinnen endgültig befreit und konnten von jetzt ab zwanglos jede beliebige Wabe belaufen. Erst jetzt schlüpften die Maden der ersten Absperrung. Damit waren die Völker während der Pflege der offenen Brut absolut weiselrichtig, d. h. weder Maden noch Königin befanden sich hinter irgendwelcher Absperrung. In keinem Fall hatten die zur Futtersaftgewinnung verwendeten Völker Schwarmlust oder auch nur den geringsten Nachschaffungstrieb.

Der Futtersaft von den Waben, auf denen die Königinnen am Mittwoch abgesperrt worden waren, wurde am darauffolgenden Montag, der von den c Donnerstagwaben » am Dienstag, und der von den " Freitagwaben ) am Mittwoch, jeweils in der Zeit von 8-14.00 Uhr, gewonnen. Da ich jede Wabe unmittelbar vor ihrer Futtersaftausbeutung aus dem Volk nahm, stammte der Futtersaft von höchstens 2 1/4-tägigen und mindestens 1-tägigen Maden. Mehrere Hilfskräfte sammelten ihn in gemeinsame eisgekühlte Glas fläschchen.

Als beste Art der Gewinnung erwies sich das Absaugen mit dem Mund in eine Glaspipette, die an einem Gummischlauch mit Holzmundstück angeschlossen war. Jede Made wurde unmittelbar vor jedem Saugakt mit Hilfe einer Umlarvnadel aus der Zelle entfernt. Während dessen war die Wabe zum Schutz gegen Austrocknung in feuchte Tücher eingewickelt, wobei nur ein kleiner Spalt zum Arbeiten frei blieb.

Die Ausbeute aus einer Wabe schwankte zwischen $1 / 2$ und $2 \mathrm{~g}$. Stets waren die a Mittwochwaben " (Montagausbeute) die schlechtesten; die "Donnerstagwaben " waren besser, und die (C Freitagwaben " (Mittwochausbeute) erwiesen sich als die besten. Das erklärte sich so, daß mit Herausnahme jeder neuen Larvenwabe aus dem Bienenvolk die zu pflegende offene Brut weniger wurde. Die verbleibenden Maden erhielten dadurch entsprechend mehr Futtersaft. Dies bestätigt einmal mehr, da $\beta$ sich die quantitative Futterversorgung der Brut bei gleicher P flegestärke des Volkes nach dessen Brutumfang richtet.

Der auf die beschriebene Weise gewonnene Futtersaft hatte einen Wassergehalt von ca. 70-72 \%. Wie schon erwähnt, beträgt nach SHuEL und Dixon (1968) der Zuckergehalt im Arbeiterinnenfuttersaft um $4 \%$, im Königinnenfuttersaft um $12 \%$. Ich brachte den gewonnenen Futtersaft unter Zugabe von $30 \%$ igem Zuckerwasser (halb Glukose, halb Fruktose) auf eine Konzentration von ca. $10 \%$ Zucker. Die Veränderung der natürlichen Diät führte ich stets erst unmittelbar vor der Verfütterung, entweder mit frisch gewonnenem oder mit höchstens 1-3 Tage in der Gefriertruhe bei $-27^{\circ} \mathrm{C}$ gespeichertem Futtersaft aus. 


\section{b) Larvenaufzucht}

\section{Versuche mit Arbeitermaden}

Zur Gewinnung des geeigneten Madenalters sperrte ich die Zuchtkönigin in der Regel am Mittwoch um 8.00 Uhr für 6 Stunden unter einen Absperrgitterrahmen auf eine helle Wabe. Am darauffolgenden Montag um 14.00 Uhr begann ich mit den jetzt 2-2 1/4-tägigen Maden die künstliche Aufzucht. Die Maden standen also noch voll in der Phase der morphologischen Umstimmbarkeit zur Königin.

Ich verwendete die amerikanische Aufzuchtmethode der regelmäßigen Larvenumbettung auf neuen Futtersaft (WEAver 1958, 1974a, Sмгтн 1959, JAY 1959 und spätere). Als Aufzuchtbehälter dienten Plastikschälchen (Schnappdeckelchen von Analysengläsern) mit $10 \mathrm{~mm}$ innerer Weite. Zuerst wurden die Maden zwecks Futterersparnis zu mehreren - und erst später, wenn sie sich gegenseitig störten - einzeln in jedem Schälchen gehalten. Alle 12 Stunden quartierte ich sie auf neuen Futtersaft um, wobei ich die Futtersaftmenge nach dem zunehmenden Appetit der heranwachsenden Maden bemaß.

Die Larvenbehälter standen in geschlossenen Glasschalen mit $98 \%$ rel. Feuchte im Brutschrank bei $35^{\circ} \mathrm{C}$. Nach dem Abkoten der Larven oder - wen dieses Ereignis nicht sichtbar wurde - nach dem Auftreten von Spinnfäden im Futter - spätestens jedoch am Ende des 10. Entwicklungstages wurden die Maden endgültig vom Futter genommen, auf einem feuchten Filterpapier flüchtig von anhaftenden Resten befreit und in pharmakologische Gelatinekapseln von $3 / 4$ oder $1 \mathrm{ccm}$ Inhalt überführt. Ich schnitt die engere Hälfte der Kapsel der Länge nach auf, um die Maden beim Einlegen nicht zu schädigen. Die Kapselenden waren zur Belüftung durchlöchert.

Gesunde Larven verpuppen sich auch ohne den Schutz der Kapseln einfach in den Aufzuchtschälchen, wenn man die letzten Futtersaftreste daraus absaugt. Ich habe aber die Kapseln vorgezogen - auch weil sich mit ihrer Hilfe die Maden besonders schonend wiegen ließen.

Zur Verpuppung setzte ich die relative Feuchte bei gleichbleibender Brutnesttemperatur auf $60 \%$ herab. Kurz vor der Imaginalhäutung entnahm ich die Puppen aus den Gelatinekapseln, bestimmte ihr Gewicht, und legte sie auf den Boden umgekippter hölzerner Königinnenschlupfkäfige, welche mit Wasser und Zuckerfutterteig versorgt waren. Alle bis zur Imaginalhäutung oder zur fertigen Imago entwickelten Tiere kamen schließlich in $70 \%$ igen Alkohol, wo sie bis zur Untersuchung ihrer Merkmale aufbewahrt wurden.

\section{Versuche mit Königinnenmaden}

In derselben Weise wie mit den altersdatierten Arbeiterlarven verfuhr ich auch mit Larven, die vom Schlupf weg königlich vorgep flegt worden waren. Im Alter von ziemlich genau 3 Tagen, d.h. nahe an der Determinationsschwelle, wurde die Hälfte der Tiere dieser Zuchtserien im Brutschrank mit Arbeiterfuttersaft künstlich aufgezogen. Die andere Hälfte ließ ich von einem normalen weiselrichtigen Bienenvolk als Arbeitermaden weiterp flegen.

Dazu brachte ich sie in der Mitte einer offenen Brutwabe in Arbeiterzellen unter, aus denen ich vorher die etwa gleichaltrigen Arbeiterinnenmaden entfernt hatte. Auf einer über die Wabe gelegten Kunststoffolie markierte ich die Lage jeder Versuchsmade, damit ich später die einzelnen Tiere nach ihrer Nummer wiederfinden konnte. Jede Larve wurde vor der Umbettung gewogen. 
Mindestens 6 Tage nach dem. Deckeln schnitt ich das Wabenstück mit den Versuchstieren aus der Brutwabe und brachte es nach vorsichtigem Entfernen der Wachsdeckel in den Brutschrank. Nach völliger Ausfärbung der Augen löste ich die Puppen aus den Zellen, wog sie und überführte sie - wie ihre manuell aufgezogenen Geschwister — einzeln in die erwähnten Schlupfkäfige, wo sie ihre letzte Häutung durchmachten.

\section{c) Merkmalsbeurteilung}

\section{Zur Beschreibung und vergleichenden Beurteilung der Aufzuchttiere} konzentrierte ich mich auf wenige, besonders markante Kastenunterschiede, wie Gewicht, Ovariolenzahl, Samenblasengröße, Ausbildung der Oberkiefer und Hinterbeine sowie Zahl der Stachelwiderhaken.

Das Puppengewicht, welches ich wenige Tage vor der erwarteten Imaginalhäutung be den bereits elfenbeinfarbenen, dunkeläugigen Tieren ermittelte, hat sich nach früheren Untersuchungen als Vergleichsgewicht bedeutend besser bewährt als das Schlupfgewicht, welches innerhalb weniger Stunden außerordentlich sehwankt (WEIss 1967).

Zur Zählung der Ovariolen präparierte ich die Eierstöcke unter Wasser aus dem Hinterleib der fertig entwickelten Tiere und fixierte sie in einer Lösung aus 80 Teilen $96 \%$ igem Alkohol und 20 Teilen $40 \%$ igem Formalin plus etwas Neutralrot zur Anfärbung. Nach einem halben Tag oder später überführte ich sie in einen Wassertropfen auf einem Objektträger mit Hohlschliff. Gezählt wurde unter dem Binokular bei 48-facher Vergrößerung unter Zuhilfenahme feinster Präpariernadeln| (Minutienstifte). Die wenigen Eischläuche der Arbeiterovarien ließen sich dabei leicht auseinandertrennen. Dagegen mußten die umfangreicheren Eierstöcke der Zwischentiere und Königinnen zuerst von den anhaftenden Häuten und Tracheen befreit werden, ehe mit einem Rasierklingensplitter unter Dagegenhalten einer Präpariernadel aus jeder Ovarhälfte an der dicksten Stelle eine Querscheibe herausgeschnitten werden konnte. Davon wurden nacheinander kleine Ovariolenbündelchen abgetrennt und gesondert ausgezählt.

Die Samenblase wurde noch in der Wasserschale gleich nach dem Freilegen bei 48-facher Vergrößerung mit Tracheenhülle gemessen. Als Meßstrecken dienten ihre mediane und laterale Ausdehnung.

Die äbrigen (äuBeren) Merkmale wurden erst nach völliger Austrocknung der Versuchstiere untersucht. Zuerst legte jch die vom Kopf abgespaltenen Oberkiefer mit der Innen fläche nach oben auf einen Objektträger, umgab sie mit einer etwa $0,3 \mathrm{~mm}$ starken. Papiermaske und deckte sie mit einem zweiten etwas kleineren Objektträgerglas ab. Bei 64-facher Vergrößerung bestimmte ich im Durchlicht die Mandibellänge $l_{\mathrm{m}}$, welche von der Mandibelspitze bis zum ventralen Wurzelgelenk reicht, und die Breite des Mandibels $b_{\mathrm{m}}$ an seiner schmalsten Stelle (Abb. 1). Gelegentlich auftretende Unebenheiten und die dichte Beborstung, besonders des Königinnenmandibels, erschwerten die Messungen, machten sie aber nicht unmöglich. Aus den beiden Meßwerten ergibt sich der Mandibelindex $l_{\mathrm{m}}: b_{\mathrm{m}}$, welcher bei der Königin relativ klein, bei der Arbeiterin größer ausfällt.

In analoger Weise, nur unter Anwendung geringerer (32-facher) Vergrößerung, bestimmte ich auch das Längen : Breitenverhältnis der Hinterbeinfersen. Der Metatarsus der Königin ist länger und schmaler als die Arbeiterferse. Bei der Messung lagen die Fersen mit der Innenseite nach oben. Die Fersenlänge $l_{\mathrm{f}}$ reicht vom freien distalen Ende der Ferse (neben der Eingelenkungsstelle der Zehenglieder) bis zum Pollenschieber der Arbeiterin oder dem königlichen Aequivalent dazu; die Breite der Ferse $b_{\mathrm{f}}$ wurde an ihrer breitesten Stelle bestimmt (Abb. 1). Bei richtiger Ausleuchtung stellte die Behaarung der Ferse kein Meßhindernis dar. Der Fersenindex $l_{\mathrm{f}}: b_{\mathrm{f}}$ ist ein gutes Vergleichsma $\beta$ für die kastenspezifische Formverschiedenheit.

Ein besonders wichtiges Kastenindiz liefert die Ausbildung der Kaukante der Mandibel. Sie ist bei der Königin durch einen deutlichen Zahn gekennzeichnet, welcher der Arbeiterin fehlt.

Ebenso aufschlußreich ist die Gestaltung der Innenseite der Hinterbeinferse. Nur die Arbeitsbiene besitzt hier eine " Bürste ), bestehend aus etwa 10 deutlich quer formierten Borstenreihen. Die Königin weist in diesem Bezirk eine gleichmäßige Behaarung auf. 

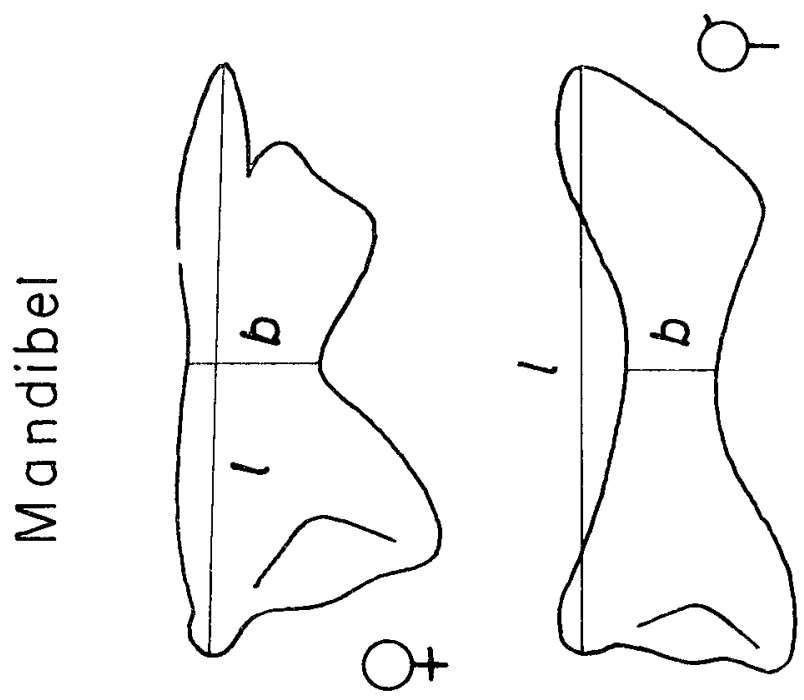

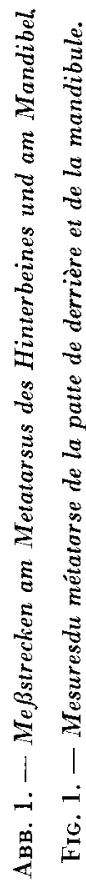

$n$
$J$
0
1
0
+0
$+\infty$
\pm
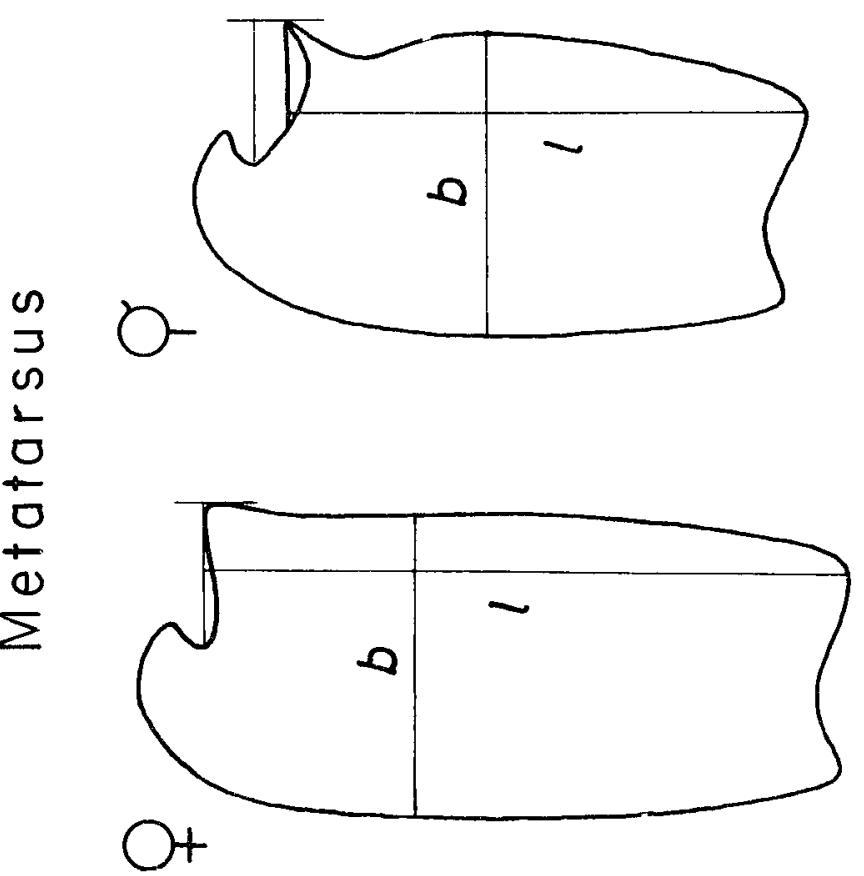
Auch das an der Außenseite der Tibia gelegene "Körbchen » findet sich nur bei der Arbeiterin. Randständige lange einwärts gekrümmte Haare begrenzen eine kahle Chitinfläche, die im unteren Teil der Tibia eine leichte Mulde bildet. Darin steht eine lange einzelne Borste. Die königliche Tibia ist dagegen über die ganze Außenfläche gleichmäBig behaart.

Die Arbeiterin besitzt außerdem am distalen Ende der Tibia den " Pollenkamm ", der aus besonders kräftigen, leicht geschwungenen in einer Reihe stehenden Borsten gebildet wird. Der Königin fehlt diese Einrichtung, oder sie ist nur andeutungsweise vorhanden.

Als letztes unterschiedliches Kastenkriterium wählte ich den Stachel. Abgesehen von kastentypischen Unterschieden in der Stärke und Krümmung, besitzt der Stachel der Arbeiterinnen auf den beiden Stechborsten etwa 9-11 deutlich erkennbare Widerhaken, während der Königinnenstachel meist nur 3-4 Widerhaken aufweist. Zur Zählung der Widerhaken zieht man die beiden Stechborsten aus der Stachelrinne und betrachtet sie unter dem Mikroskop in einem Tropfen Terpineol zwischen Objektträger und Deckglas bei wechselweiser 300und 600-facher Vergrößerung.

\section{d) Bienenmaterial, Zeitpunkt der Versuche und personelle Hilfe}

Alle Versuche wurden in Erlangen mit Carnica-Bienen durchgeführt. Die wesentlichen Laboraufzuchten liefen von Anfang Juni bis Mitte August 1973. Zur Gewinnung des Arbeiterfuttersaftes hat sich unsere Laborbelegschaft tatkräftig eingesetzt. Bei der Präparation der Aufzuchtprodukte während der folgenden Wintermonate stand mir, wie schon bei früheren Untersuchungen, Anna Tschakowsky gewissenhaft zur Seite. Allen Beteiligten möchte ich auch hier meinen Dank abstatten.

\section{III. - VERSUCHSERGEBNISSE}

\section{a) Umfang und Verlauf der Versuche}

Bei der künstlichen Aufzucht von Arbeiterinnen - und Königinnenmaden im Brutschrank gibt es Ausfälle. Wenn man den Erfolgsgrad der Aufzuchtbemühungen beurteilen will, muß man den Umfang der Individuensterblichkeit während der Larven- und Puppenentwicklung kennen. Ich habe deshalb für alles Versuchsmaterial die Ausfälle auf jedem Entwicklungsstadium und die Überlebensquoten zahlenmäßig in Tabelle 1 festgehalten. Die Versuchsgruppen I, II, III und IV sind nach der Kastenzugehörigkeit der Versuchsmaden und der verabreichten Larvennahrung verschieden. Sie setzen sich aus mehreren kleinen, in den verschiedenen Versuchswochen durchgeführten Versuchsansätzen zusammen, was in der Tabelle nicht berücksichtigt ist. Die kleinen Versuchsansätze waren nötig, weil der frisch zu verfütternde Arbeiterfuttersaft immer nur in begrenztem Ausmaß zur Verfügung stand. Dadurch konnte nur eine relativ kleine Anzahl von Maden gleichzeitig gefüttert werden. 
ERNÄHRUNG DER WEIBLICHEN BIENENLARVE

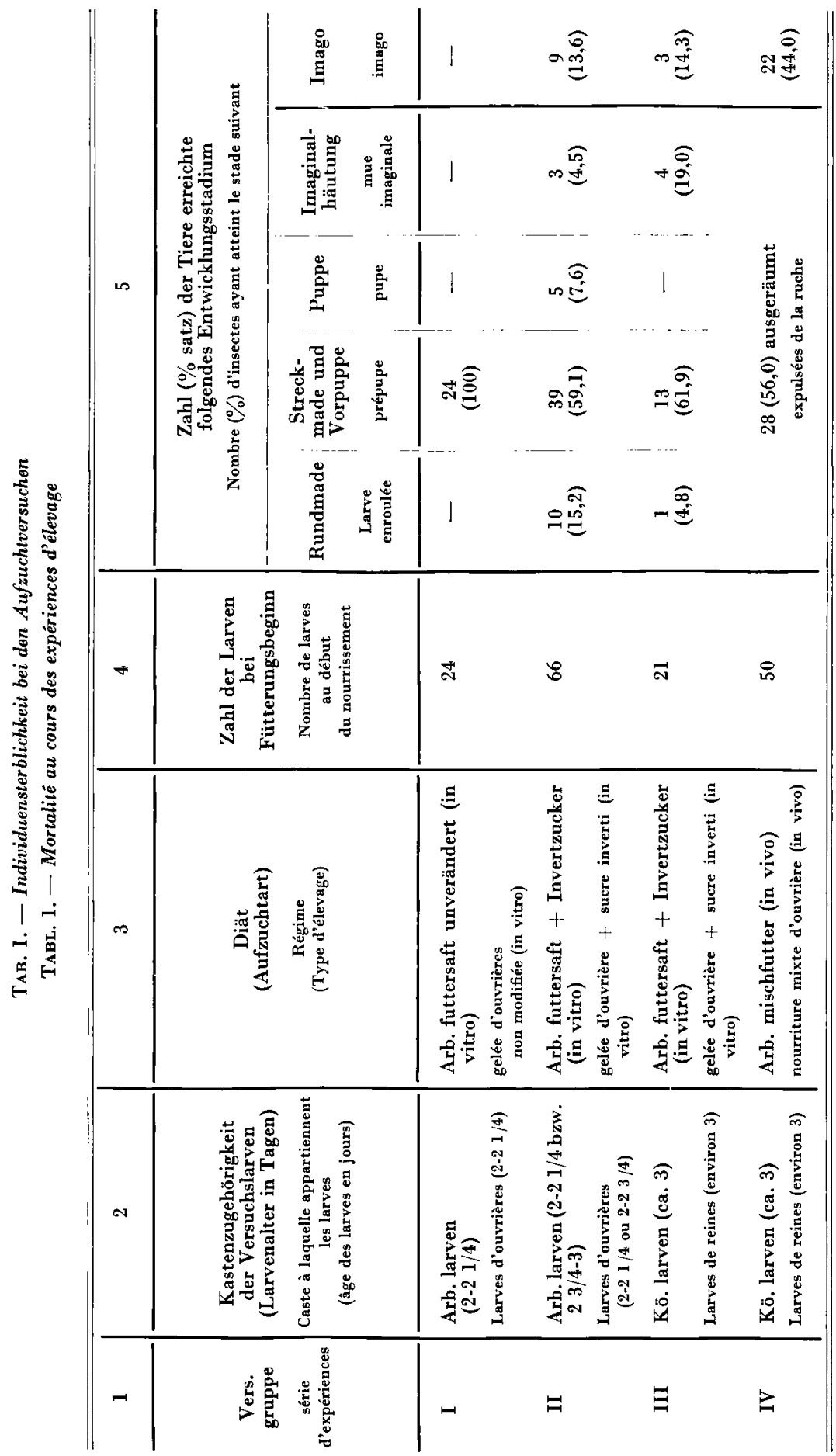


Versuchsgruppe I bestätigt die bisherige Erfahrung, daß sich mit reinem Arbeiterfuttersaft ohne Zuckerzusatz keine Made zur Puppe verwandelt.

In der II. und III. Versuchsgruppe mit Arbeiterinnen- bzw. Königinnenmaden als Ausgangsmaterial für manuelle Aufzucht führte die Versorgung mit gesüßtem Arbeiterfuttersaft bei einem Teil der Tiere zur Verpuppung und Weiterentwicklung zur Imago. Die Zahl der erreichten Adulten oder Fast-Adulten betrug bei den Königinnenlarven (Gruppe III) $33,3 \%$, bei den Arbeiterinnenlarven (Gruppe II) nur 18,1\%.

In Versuchsgruppe IV wurden die verwendeten Königinnenmaden nicht wie in Gruppe III im Labor (mit Arbeiterfuttersaft) aufgezogen, sondern nach der Überführung in Arbeiterzellen eines weiselrichtigen Bienenvolkes von regulären Ammen (vermutlich mit Mischfutter) weitergepflegt. 44,0\% entwickelten sich zu Imagines. Bei einem zweiten, in der Tabelle nicht berücksichtigten Versuch räumten die Bienen sämtliche in die Arbeiterzellen überführten Königinnenmaden aus.

Der Prozentsatz der fertig entwickelten Tiere bei den manuell aufgezogenen Larven erscheint gering. Andere Autoren haben mit naturbelassenen oder veränderten (Königinnen-) Futtersäften bessere Ergebnisse erzielt - wobei sich allerdings in jedem Fall nur ein Teil der Tiere zu wirklichen Königinnen entwickelte. Wenn man von den vielerlei möglichen technischen Unzulänglichkeiten in der Aufzucht absieht, möchte man in erster Linie eine gewisse Unstimmigkeit zwischen der Beschaffenheit der Larven und der Futterart für die Ausfälle verantwortlich machen. Ganz allgemein läuft die Entwicklung der Larven bei der Laboraufzucht leichter in Richtung Arbeitsbiene als in Richtung Königin. Dabei hat es den Anschein, daß die Ausbildung von Zwischentieren Entwicklungsschwierigkeiten bereitet und daß ein gewisser Anteil der Ausfälle gerade hierauf zurückzuführen ist. Möglicherweise war die Tendenz zu Zwischentieren in meinen Versuchen besonders groß.

\section{b) Beurteilung der Aufzuchtprodukte}

Alle in vitro und in vivo aufgezogenen Versuchstiere sind in Tabelle 2 einzeln aufgeführt und nach ihrer Kastenzugehörigkeit beurteilt. Dabei ließen sich Gewichte, Ovariolen, Mandibel- und Fersenindizes sowie Stachelwiderhaken in Zahlen fassen. Die übrigen Untersuchungsmerkmale wurden nach dem Grad ihrer kastenspezifischen Ausbildung in ein 4-klassiges Bewertungsschema eingestuft. Es bedeutet « $K$ » königinnentypisch, « $A$ » einwand-

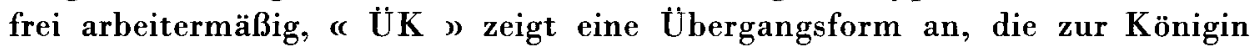
tendiert, “ ÜA » weist mehr zur Arbeiterin hin und “ Ü » allein besagt, daß das Merkmal ziemlich ausgeglichen zwischen der königlichen und arbeiterhaften Ausbildung rangiert. 
Im einzelnen gilt folgendes Beurteilungsschema :

Mandibelkante

$\mathrm{K}=$ Zahn durch Einkerbung deutlich abgesetzt (Abb. 2).

ÜK $=$ Kerbe reduziert.

$\dot{\mathbf{U}}=$ Kerbe nur noch angedeutet oder fehlend bei abgesetzter Mandibelspitze (Abb. 2)

$\ddot{\mathrm{U} A}=$ keine Kerbe, Mandibelkante nicht abgesetzt, Mandibel spitzig, oft mit etwas aufgebogenem oberen Rand (Abb. 2)

$\mathrm{A}=$ Mandibel löffelförmig gerundet (Abb. 2)

Bürste

$K=$ Bürste fehlt (Abb. 3)

ÜK $=$ unterste Borsten verstärkt

$\dot{\mathrm{U}}=$ Borstenreihen beginnen sich zu ordnen, unterste bereits klar formiert (Abb. 3)

$\ddot{\mathrm{U} A}=$ Ordnung der Borstenreihen fortgeschritten

$A=$ Borstenreihen deutlich ausgebildet (Abb. 3)

Kamm

$\mathbf{K}=$ so gut wie nicht vorhanden

$\ddot{\mathrm{U} K}=$ Kammborsten wenig verstärkt

$\dot{\mathrm{U}}=$ Kammborsten stärker ausgeprägt

$\ddot{U} A=$ Kammborsten zinkenartig, wenn auch noch nicht arbeiter-mäßig ausgeformt

$A=$ deutlich gezinkter, starker Kamm

Körbchen

$\mathbf{K}=$ Körbchen fehlt

ÜK = Behaarung der Körbchenfläche wird schütter

$\dot{\mathrm{U}}=$ Randhaare werden länger, Innenbehaarung nimmt weiter ab

ÜA = Haarumrandung klar ausgebildet, aber noch mehrere Innenborsten vorhanden

$\mathbf{A}=$ glatt, mit typischer Randbehaarung, großes Kontakthaar in der Mitte des Körbchens deutlich hervortretend

Während diese klassenmäßig erfaßten Merkmale die graduelle Zugehörigkeit eines Versuchstiers zu einer der beiden weiblichen Bienenkasten in Tabelle 2 sofort ausweisen, ist für die zahlenmäßig erfaßten Kriterien eine Vergleichsmöglichkeit mit typisch ausgeprägten Königinnen und Arbeiterinnen notwendig. Deshalb sind zu Beginn der Tabelle die Minimum- und Maximumwerte der gemessenen bzw. gezählten Untersuchungsmerkmale neben den Symbolen der nicht meßbaren für mehrere typische Königinnen und Arbeiterinnen angegeben. Königinnen und Arbeiterinnen stammen aus dem Volk, welches auch den Zuchtstoff für die Mehrzahl der Laboraufzuchten geliefert hat. Die Königinnen wurden in einem optimalen (weisellosen) Pflegevolk erbrütet.

Die wichtigste Aussage der Tabelle findet sich in der II. Versuchsgruppe. Dort wird deutlich, daß es möglich ist, junge Arbeiterinnenmaden im Labor mit ausschließlicher Verabreichung von Arbeiterfuttersaft zu adulten Tieren mit eindeutig königlichen Merkmalen aufzuziehen. Das gelang (in allen Merkmalen) überzeugend aber nur bei 2 von 12 Tieren, die das Endstadium der Entwicklung erreichten (Nr. 9 und 12)*. Dabei scheint eine Abhängigkeit

\footnotetext{
* Die interessanten Übergangsformen des Zwischentieres Nr. 9 sind in den Abbildungen 2,3 und 4 wiedergegeben.
} 
TAB. 2. - Beschaffenh

TABL. 2. - Qualité des Insect

\begin{tabular}{|c|c|c|c|c|c|c|c|}
\hline 1 & 2 & 3 & 4 & & 5 & & 6 \\
\hline $\begin{array}{c}\text { Vers. } \\
\text { gruppe } \\
\text { Série } \\
\text { d'expérience }\end{array}$ & $\begin{array}{c}\text { Art der } \\
\text { Versuche } \\
\text { Type } \\
\text { d'expérience }\end{array}$ & $\begin{array}{c}\text { Tier } \\
\text { Nr. } \\
\text { Insecte } \\
n^{\circ}\end{array}$ & $\begin{array}{c}\text { Larv. } \\
\text { alter } \\
\text { bei } \\
\text { Vers. } \\
\text { beg. } \\
\text { in } \\
\text { Tagen } \\
\text { Age des larves } \\
\text { en jours } \\
\text { au début } \\
\text { de l'expérience }\end{array}$ & $\begin{array}{c}\text { Rund- } \\
\text { made } \\
\text { bei } \\
\text { Vers. } \\
\text { beg. } \\
\\
\text { larve enroulée } \\
\text { au début } \\
\text { de l'expérience }\end{array}$ & $\begin{array}{c}\text { ewicht in mg } \\
\text { Streck- } \\
\text { made } \\
\text { beim } \\
\text { Ver- } \\
\text { schulen } \\
\text { Poids en mg } \\
\text { prépupe } \\
\text { au début } \\
\text { de l'expérience }\end{array}$ & $\begin{array}{l}\text { Puppe } \\
\text { pupe }\end{array}$ & $\begin{array}{c}\text { Entwick- } \\
\text { lungs- } \\
\text { zeit } \\
\text { in } \\
\text { Tagen } \\
\text { Durée de } \\
\text { développeme }\end{array}$ \\
\hline I & $\begin{array}{l}\text { in vivo aufgezog. } \\
\text { typ. Arbeiterinnen } \\
\text { (17 Tiere) } \\
\text { ouvrières typiques élevées } \\
\text { in vivo (17 insectes) } \\
\text { in vivo aufgezog. } \\
\text { typ. Königinnen } \\
\text { (27 Tiere) } \\
\text { Reines typiques élevées in } \\
\text { vivo (27 insectes) }\end{array}$ & & & & & $\begin{array}{r}137 \\
-151\end{array}$ & $20-21$ \\
\hline II & $\begin{array}{l}\text { Arbeiterlarven } \\
\text { in vitro } \\
\text { gefüttert mit } \\
\text { Arb. futtersaft } \\
+ \text { Invertzucker } \\
\text { Larves d'ouvrières nourries } \\
\text { in vitro avec de la gelée } \\
\text { d'ouvrière + suere inverti }\end{array}$ & $\begin{array}{r}1 \\
2 \\
3 \\
4 \\
5 \\
6 \\
7 \\
8 \\
9 \\
10 \\
11 \\
12\end{array}$ & $\begin{array}{l}2-21 / 4 \\
23 / 4-3\end{array}$ & $\begin{array}{r}3,3 \\
4,8 \\
4,6 \\
5,2 \\
5,1 \\
4,6 \\
3,9 \\
4,8 \\
5,0 \\
13,9 \\
11,0 \\
17,5\end{array}$ & $\begin{array}{l}181 \\
204 \\
185 \\
207 \\
214 \\
222 \\
159 \\
207 \\
265 \\
269 \\
259 \\
291\end{array}$ & $\begin{array}{l}143 \\
165 \\
146 \\
156 \\
171 \\
181 \\
138 \\
187 \\
215 \\
208 \\
182 \\
248\end{array}$ & $\begin{array}{l}21 \\
20 \\
20 \\
21 \\
20 \\
20 \\
21 \\
21 \\
18 \\
20 \\
21 \\
16\end{array}$ \\
\hline III & $\begin{array}{l}\text { König larven } \\
\text { in vitro } \\
\text { gefüttert mit } \\
\text { Arb futtersaft } \\
\text { + Invertzucker } \\
\text { Larves de reines nourries in } \\
\text { vitro avec de la gelée d'ou- } \\
\text { vrière et du sucre inverti }\end{array}$ & $\begin{array}{l}1 \\
2 \\
3 \\
4 \\
5 \\
6 \\
6\end{array}$ & ca. 3 & $\begin{array}{l}11,0 \\
12,8 \\
11,1 \\
15,0 \\
13,5 \\
14,9 \\
10,7\end{array}$ & $\begin{array}{l}311 \\
309 \\
300 \\
\mathbf{3} 06 \\
314 \\
338 \\
266\end{array}$ & $\begin{array}{l}259 \\
248 \\
249 \\
258 \\
264 \\
285 \\
212\end{array}$ & $\begin{array}{l}15 \\
16 \\
16 \\
16 \\
16 \\
16 \\
16\end{array}$ \\
\hline IV & $\begin{array}{l}\text { König. larven } \\
\text { in vivo } \\
\text { weitergefüttert } \\
\text { als } \\
\text { Arbeiterlarven } \\
\text { Larves de reines nourries in } \\
\text { vivo comme des larves } \\
\text { d'ouvrières }\end{array}$ & $\begin{array}{l}1-13 \\
14 \\
15 \\
16 \\
17 \\
18 \\
19 \\
20 \\
21 \\
22\end{array}$ & ca. 3 & $\begin{array}{r}<10,0 \\
10,9 \\
13,2 \\
12,0 \\
10,5 \\
11,0 \\
11,5 \\
11,5 \\
12,5 \\
11,9\end{array}$ & & $\begin{array}{r}96 \\
-140 \\
134 \\
137 \\
128 \\
94 \\
139 \\
104 \\
106 \\
96 \\
132\end{array}$ & $\begin{array}{c}20-21 \\
20 \\
20 \\
20 \\
19 \\
19 \\
18 \\
18 \\
18 \\
19\end{array}$ \\
\hline
\end{tabular}
a) nicht gefunden, b) $\begin{gathered}\text { verkümmert, c) } \\ \text { mal développé }\end{gathered}$
non trouvé non dénombrable, tissu spongieux perdu 
Versuchstiere.

ilisés pour les expêriences.

\begin{tabular}{|c|c|c|c|c|c|c|c|c|}
\hline & & & & & & & & 11 \\
\hline $\begin{array}{r}\text { Reprod } \\
\text { sys } \\
\text { Système } r\end{array}$ & $\begin{array}{l}\text { stions- } \\
\text { roducteur }\end{array}$ & $\begin{array}{l}\mathrm{Ma} \\
\mathrm{Ma}\end{array}$ & $\begin{array}{l}\text { bel } \\
\text { ule }\end{array}$ & $\begin{array}{r}\text { Met } \\
\text { Mé }\end{array}$ & $\begin{array}{l}\text { rsus } \\
\text { rse }\end{array}$ & & & $\begin{array}{c}\text { Zahl } \\
\text { der } \\
\text { Stache } \\
\text { häkche }\end{array}$ \\
\hline $\begin{array}{l}\text { Ovari- } \\
\text { olen- } \\
\text { zahl } \\
\text { bre d'ove- } \\
\text { rioles }\end{array}$ & $\begin{array}{c}\text { Samen- } \\
\text { blasen- } \\
\text { größe } \\
\text { i. Teil- } \\
\text { strichen } \\
\text { Taille de } \\
\text { la sperma- } \\
\text { thèque }\end{array}$ & $\begin{array}{c}\text { Mand. } \\
\text { index } \\
\text { li/re } \\
\text { Index } \\
\text { mandibu- } \\
\text { laire }\end{array}$ & $\begin{array}{c}\text { Kau- } \\
\text { kante } \\
\text { li/re } \\
\text { Arête } \\
\text { masti- } \\
\text { catoire }\end{array}$ & $\begin{array}{c}\text { Tarsal- } \\
\text { index } \\
\text { li/re } \\
\text { Index } \\
\text { tarsal }\end{array}$ & $\begin{array}{c}\text { Bürste } \\
\text { li/re } \\
\text { Brosse }\end{array}$ & $\begin{array}{c}\text { Pollen- } \\
\text { kamm } \\
\text { li/re } \\
\text { Peigne } \\
\text { à pollen }\end{array}$ & $\begin{array}{c}\begin{array}{c}\text { Körb- } \\
\text { chen }\end{array} \\
\text { li/re } \\
\text { Corbeille }\end{array}$ & $\begin{array}{l}\text { li/re } \\
\text { Nombre de } \\
\text { croebets de } \\
\text { l'aiguillon }\end{array}$ \\
\hline $2-11$ & $\begin{array}{r}\mid 6-7 / \\
-\mid 4-6 /\end{array}$ & $4,4-5,1$ & $\mathbf{A} / \mathbf{A}$ & $1,6-1,8$ & A/A & $\mathbf{A} / \mathbf{A}$ & $\mathbf{A} / \mathbf{A}$ & $9-10$ \\
\hline $\begin{array}{r}323 \\
-386\end{array}$ & $\begin{array}{r}\mid 45-52 / \\
-/ 47-50 /\end{array}$ & $3,3-3,9$ & $\mathrm{~K} / \mathrm{K}$ & $1,9-2,3$ & $\mathbf{K} / \mathbf{K}$ & $\mathbf{K} / \mathbf{K}$ & $\mathrm{K} / \mathrm{K}$ & $3-4$ \\
\hline $\begin{array}{r}5 \\
14 \\
7 \\
22 \\
10\end{array}$ & $\begin{array}{c}14: 13 \\
16: 16 \\
\text { a) } \\
\text { e) } \\
13: 13\end{array}$ & $\begin{array}{l}4,9 / 5,2 \\
5,1 / 4,3 \\
4,5 / 4,5 \\
5,2 / 5,1 \\
4,8 / 5,1\end{array}$ & $\begin{array}{l}\mathbf{A} / \mathbf{A} \\
\mathbf{A} / \mathbf{A} \\
\mathbf{A} / \mathbf{A} \\
\mathbf{A} / \mathbf{A} \\
\mathbf{A} / \mathbf{A}\end{array}$ & $\begin{array}{l}1,6 / 1,6 \\
1,8 / 1,8 \\
1,7 / 1,6 \\
1,8 / 1,8 \\
1,8 / 1,8\end{array}$ & $\begin{array}{l}\mathbf{A} / \mathbf{A} \\
\mathbf{A} / \mathbf{A} \\
\mathbf{A} / \mathbf{A} \\
\mathbf{A} / \mathbf{A} \\
\mathbf{A} / \mathbf{A}\end{array}$ & $\begin{array}{l}\text { A/A } \\
\text { A/A } \\
\text { A/A } \\
\text { A/A } \\
\text { A/A }\end{array}$ & $\begin{array}{l}\mathbf{A} / \mathbf{A} \\
\mathbf{A} / \mathbf{A} \\
\mathbf{A} / \mathbf{A} \\
\mathbf{A} / \mathbf{A} \\
\mathbf{A} / \mathbf{A}\end{array}$ & $\begin{array}{c}10 / 10 \\
10 / 10 \\
10 / 10 \\
9 / 9 \\
7 / 7\end{array}$ \\
\hline $\begin{array}{c}11 \\
15 \\
14 \\
57 \\
\text { c) } \\
4 \\
333\end{array}$ & $\begin{aligned} & 15: 14 \\
& 5: 5 \\
& 13: 13 \\
& 38: 35 \\
& 18: 18 \\
& 15: 15 \\
& 46: 40\end{aligned}$ & $\begin{array}{l}5,2 / 4,4 \\
4,4 / 4,6 \\
4,3 / 4,7 \\
4,4 / 4,3 \\
4,6 / 4,5 \\
4,5 / 4,7 \\
4,1 / 4,1\end{array}$ & $\begin{array}{l}\mathbf{A} / \mathbf{U}^{2} \mathbf{A} \\
\mathbf{U} \mathbf{A} / \mathbf{A} \\
\mathbf{A} / \mathbf{U} \mathbf{A} \\
\mathbf{U} \mathbf{A} / \dot{U}^{2} \\
\mathbf{A} / \mathbf{A} \\
\mathbf{A} / \mathbf{A} \\
\mathbf{K} / \mathbf{K}\end{array}$ & $\begin{array}{l}1,8 / 1,8 \\
1,8 / 1,8 \\
1,6 / 1,6 \\
1,7 / 1,8 \\
1,8 / 1,8 \\
\text { b) } \\
\text { b) }\end{array}$ & $\begin{array}{l}\mathbf{A} / \mathbf{A} \\
\mathbf{A} / \mathbf{A} \\
\mathbf{A} / \mathbf{A} \\
\mathbf{U} / \mathbf{U}^{2} \\
\mathbf{A} / \mathbf{A} \\
\mathrm{b}) \\
\mathrm{b})\end{array}$ & $\begin{array}{l}\mathbf{A} / \mathbf{A} \\
\mathbf{A} / \mathbf{A} \\
\mathbf{A} / \mathbf{A} \\
\mathbf{U}^{2} / \dot{U} \\
\mathbf{A} / \mathbf{A} \\
\mathbf{A} / \mathbf{A} \\
\mathbf{K} / \mathbf{K}\end{array}$ & $\begin{array}{l}\mathbf{A} / \mathbf{A} \\
\mathbf{A} / \mathbf{A} \\
\mathbf{A} / \mathbf{A} \\
\dot{U} / \dot{U} \\
\mathbf{A} / \mathbf{A} \\
\mathbf{A} / \mathbf{A} \\
\mathbf{K} / \mathbf{K}\end{array}$ & $\begin{array}{c}9 / 9 \\
10 / 10 \\
8 / 8 \\
7 / 8 \\
10 / 10 \\
9 / 10 \\
5 / 6\end{array}$ \\
\hline $\begin{array}{l}374 \\
343 \\
343 \\
364 \\
349\end{array}$ & $\begin{array}{l}45: 44 \\
45: 43 \\
46: 41 \\
42: 40 \\
46: 41\end{array}$ & $\begin{array}{l}\mathbf{3}, 9 / \mathbf{3}, 7 \\
\mathbf{3}, 8 / \mathbf{3}, 7 \\
3,9 / 3,7 \\
\mathbf{3}, 9 / \mathbf{3}, \mathbf{8} \\
\mathbf{3 , 9 / 3 , 5}\end{array}$ & $\begin{array}{l}\mathbf{K} / \mathbf{K} \\
\mathbf{K} / \mathbf{K} \\
\mathbf{K} / \mathbf{K} \\
\mathbf{K} / \mathbf{K} \\
\mathbf{K} / \mathbf{K}\end{array}$ & $\begin{array}{c}\text { b) } / 1,8 \\
1,9 / 1,9 \\
1,9 / b) \\
\text { b) } \\
2,0 / 2,0\end{array}$ & $\begin{array}{c}\text { b)/K } \\
\mathbf{K} / \mathbf{K} \\
\mathbf{U} \mathbf{K} / \mathbf{b}) \\
\mathbf{b}) \\
\mathbf{K} / \mathbf{K}\end{array}$ & $\begin{array}{c}\text { b) } / \mathbf{U} \mathbf{K} \\
\mathbf{K} / \mathbf{K} \\
\mathbf{K} / \mathbf{b}) \\
\mathbf{b}) \\
\mathbf{K} / \mathbf{K}\end{array}$ & $\begin{array}{c}\text { b) } / \mathbf{U} \mathbf{K} \\
\mathbf{K} / \mathbf{K} \\
\left.\mathbf{K} / \mathbf{b}^{\mathbf{b}}\right) \\
\mathbf{b}) \\
\mathbf{K} / \mathbf{K}\end{array}$ & $\begin{array}{l}5 / 5 \\
5 / 5 \\
\text { d) } \\
6 / 6 \\
4 / 4\end{array}$ \\
\hline $\begin{array}{l}331 \\
319\end{array}$ & $\begin{array}{l}42: 40 \\
42: 36\end{array}$ & $\begin{array}{l}3,6 / 3,9 \\
3,5 / 3,4\end{array}$ & $\begin{array}{l}\mathbf{K} / \mathbf{K} \\
\mathbf{K} / \mathbf{K}\end{array}$ & $\begin{array}{c}1,8 / \mathrm{b}) \\
b)\end{array}$ & $\begin{array}{c}\left.\mathbf{K} /{ }^{\mathbf{b}}\right) \\
h)\end{array}$ & $\underset{b}{K} / \mathbf{K}$ & $\underset{\mathrm{b})}{\mathbf{K} / \mathbf{K}}$ & $\begin{array}{l}4 / 4 \\
5 / 5\end{array}$ \\
\hline $2-19$ & $\begin{array}{r}|6-7| \\
-|4-7|\end{array}$ & $4,3-5,0$ & $\mathbf{A} / \mathbf{A}$ & $1,7-1,8$ & $\mathbf{A} / \mathbf{A}$ & $\mathbf{A} / \mathbf{A}$ & $\mathbf{A} / \mathbf{A}$ & \\
\hline $\begin{array}{l}2 \\
2 \\
8\end{array}$ & $\begin{array}{l}6: 5 \\
6: 4 \\
6: 4\end{array}$ & $\begin{array}{l}4,5 / 4,9 \\
4,8 / 4,5 \\
4,8 / 4,9\end{array}$ & $\begin{array}{l}\mathbf{A} / \mathbf{A} \\
\mathbf{A} / \mathbf{A} \\
\mathbf{A} / \mathbf{A}\end{array}$ & $\begin{array}{l}1,7 / 1,7 \\
1,6 / 1,7 \\
1,9 / 1,8\end{array}$ & $\begin{array}{l}\mathbf{A} / \mathbf{A} \\
\mathbf{A} / \mathbf{A} \\
\mathbf{A} / \mathbf{A}\end{array}$ & $\begin{array}{l}\mathbf{A} / \mathbf{A} \\
\mathbf{A} / \mathbf{A} \\
\mathbf{A} / \mathbf{A}\end{array}$ & $\begin{array}{l}\mathbf{A} / \mathbf{A} \\
\mathbf{A} / \mathbf{A} \\
\mathbf{A} / \mathbf{A}\end{array}$ & \\
\hline $\begin{array}{r}31 \\
66 \\
141 \\
163 \\
233 \\
260\end{array}$ & $\begin{array}{l}5: 4 \\
7: 5 \\
5: 4 \\
7: 5 \\
c) \\
7: 6\end{array}$ & $\begin{array}{l}4,9 / 4,8 \\
4,8 / 4,6 \\
4,9 / 4,9 \\
4,8 / 4,3 \\
4,8 / 4,3 \\
4,6 / 4,8\end{array}$ & $\begin{array}{c}\mathbf{A} / \mathbf{A} \\
\mathbf{A} / \mathbf{A} \\
\mathbf{A} / \mathbf{U} \mathbf{A} \\
\text { UA/A } \\
\mathbf{A} / \mathbf{U} \mathbf{A} \\
\text { UA/A }\end{array}$ & $\begin{array}{l}1,7 / 1,6 \\
1,7 / 1,7 \\
1,6 / 1,6 \\
1,7 / 1,8 \\
1,7 / 1,8 \\
1,7 / 1,7\end{array}$ & $\begin{array}{l}\mathbf{A} / \mathbf{A} \\
\mathbf{A} / \mathbf{A} \\
\mathbf{A} / \mathbf{A} \\
\mathbf{A} / \mathbf{A} \\
\mathbf{A} / \mathbf{A} \\
\mathbf{A} / \mathbf{A}\end{array}$ & $\begin{array}{l}\mathbf{A} / \mathbf{A} \\
\mathbf{A} / \mathbf{A} \\
\mathbf{A} / \mathbf{A} \\
\mathbf{A} / \mathbf{A} \\
\mathbf{A} / \mathbf{A} \\
\mathbf{A} / \mathbf{A}\end{array}$ & $\begin{array}{l}\mathbf{A} / \mathbf{A} \\
\mathbf{A} / \mathbf{A} \\
\mathbf{A} / \mathbf{A} \\
\mathbf{A} / \mathbf{A} \\
\mathbf{A} / \mathbf{A} \\
\mathbf{A} / \mathbf{A}\end{array}$ & \\
\hline
\end{tabular}



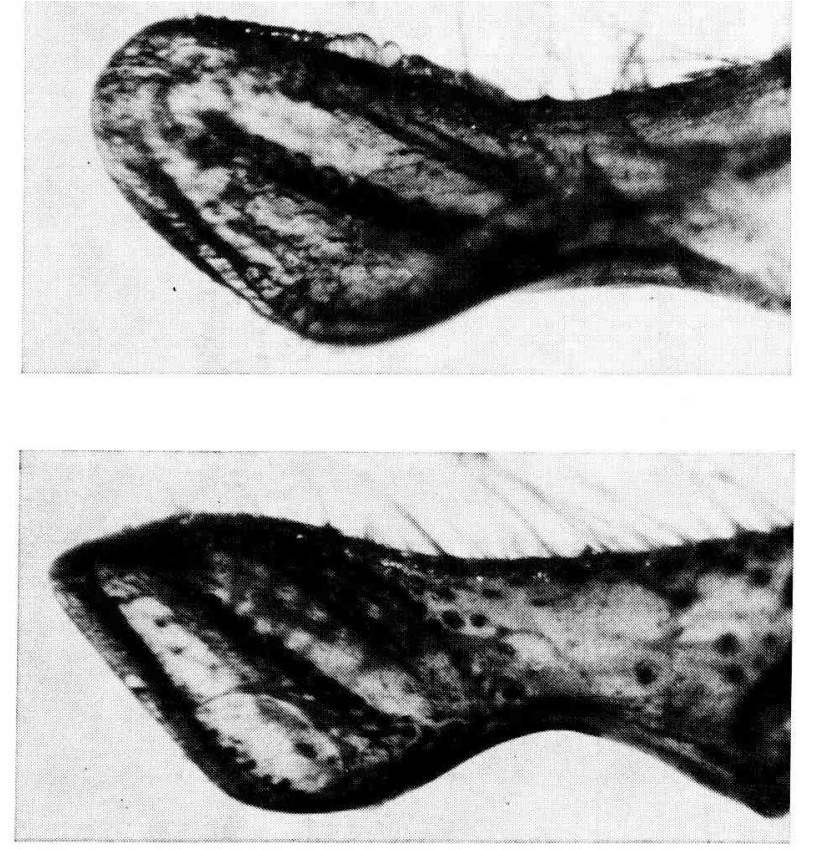

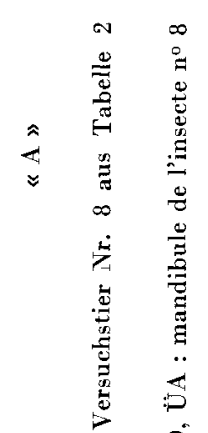
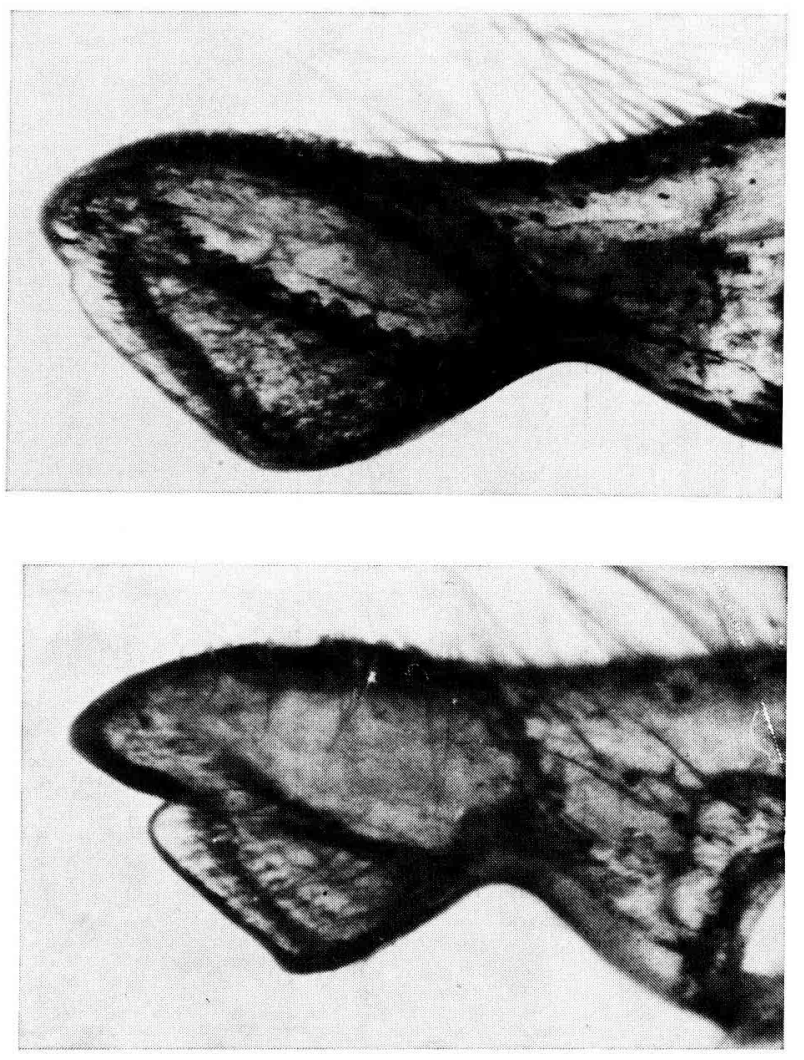

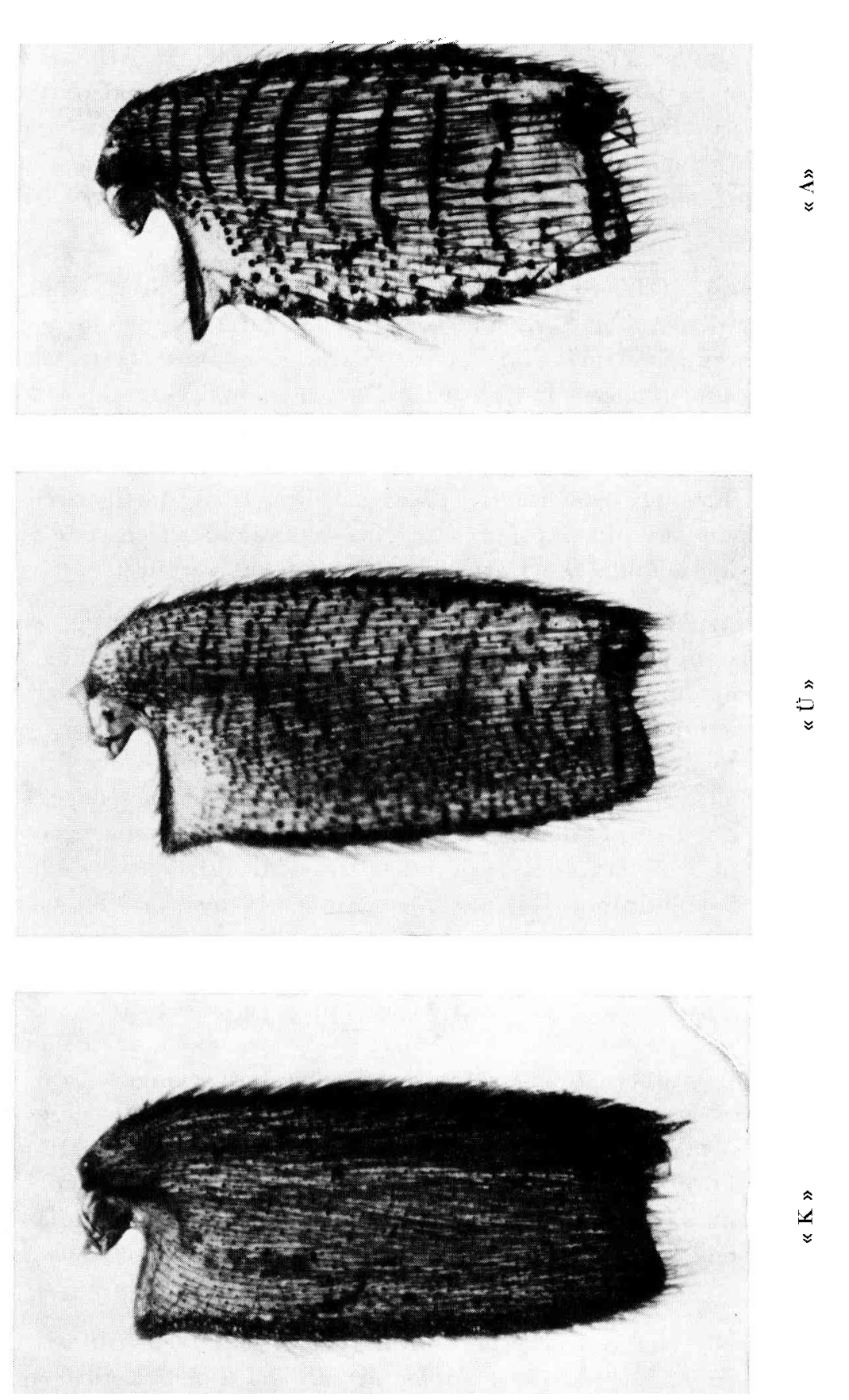

$=$

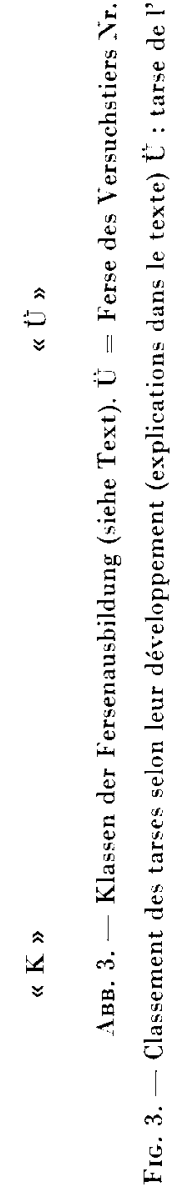


von der Menge des aufgenommenen Futters zu bestehen. In meinen Versuchen zeigten nur Tiere, die ein Streckmadengewicht von über $250 \mathrm{mg}$ erreichten, in der Adultausbildung eindeutig königliche Züge. Leichtere Streckmaden und ausnahmsweise auch schwerere (Nr. 10 und 11) wurden zu Arbeiterinnen. Auffallenderweise hatten fast alle diese Tiere gegenüber normalen Arbeitsbienen vergrößerte Samenblasen, aber nicht auch vermehrte Eischläuche. Mitunter war die Stachelhäkchenzahl bei sonst normalen Arbeitsbienen verringert, selten zeigte eine Kaulade geringfügige Abweichung vom Arbeitertyp. Die Entwicklungszeit war in keinem dieser Fälle verkürzt.

Versuchsgruppe III und IV sind vergleichend zu betrachten. Königliche Maden aus der gleichen Zuchtserie und vom gleichen Alter ergaben bei manueller Weiterfütterung mit Arbeiterfuttersaft typische Königinnen (III), während sie sich, von Ammenbienen in Arbeiterzellen eines weiselrichtigen Volkes (vermutlich mit Arbeitermischfutter) weitergefüttert, ganz vorwiegend zu Arbeiterinnen entwickelten (Versuchsgruppe IV). Einige Tiere hatten aber erheblich vermehrte Ovariolen ( $\mathrm{Nr}$. 17-22), während eigenartigerweise die Samenblase in keinem Fall vergrößert war. Nur selten fanden sich erste Spuren einer Mandibelabweichung. Die Entwicklung war etwas verkürzt.

Im Zusammenhang mit diesem Versuchsergebnis ist wichtig, daß in beiden Versuchsgruppen Tiere verglichen wurden, die aus ungefähr gleich großen Maden hervorgingen. Gleichaltrige Königinnenmaden aus derselben Zuchtserie können nämlich in der ersten Zeit ihres Larvendaseins große Gewichtsunterschiede aufweisen. So schwankten die Gewichte der 3-tägigen Königinnenlarven aus zwei zur gleichen Zeit in verschiedenen Pflegevölkern durchgeführten Zuchten in einem Fall zwischen 5,1 und 16,9 und im anderen zwischen 2,8 und 15,7 mg. Ich verwendete zu den Aufzuchtversuchen im Brutschrank (Versuchsgruppe III) nur Larven über $10 \mathrm{mg}$. Zur Weiterpflege im Volk als Arbeiterinnenlarven (in Versuchsgruppe IV) dienten dagegen auch leichtere Tiere. Es schien, als ob die Ammen diese lieber in Pflege nehmen würden als die schwereren. Aus ihnen entwickelten sich aber stets ganz eindeutige Arbeitsbienen (Nr. 1-13). Vermehrte Ovariolenzahlen traten nur bei Tieren auf, diẹ aus über $10 \mathrm{mg}$ schweren Maden hervorgingen. Nur diese (Nr. 14-22) kann man mit den Tieren in Versuchsgruppe III vergleichen. Damit fallen Bedenken weg, daß die manuell mit Arbeiterfuttersaft aufgezogenen und zu Königinnen avancierten Larven der Versuchsgruppe III zu Versuchsbeginn in stärkerem Maße königlich vorgeprägt gewesen sein könnten als die im Volk mit Arbeitermischfutter ernährten Maden der Versuchsgruppe IV.

Bestehen bleibt der Finwand, daß die in den Arbeiterzellen in vivo aufgezogenen Larven kleinere Tiere ergaben als die im Labor gefütterten (s. Diskussion). 


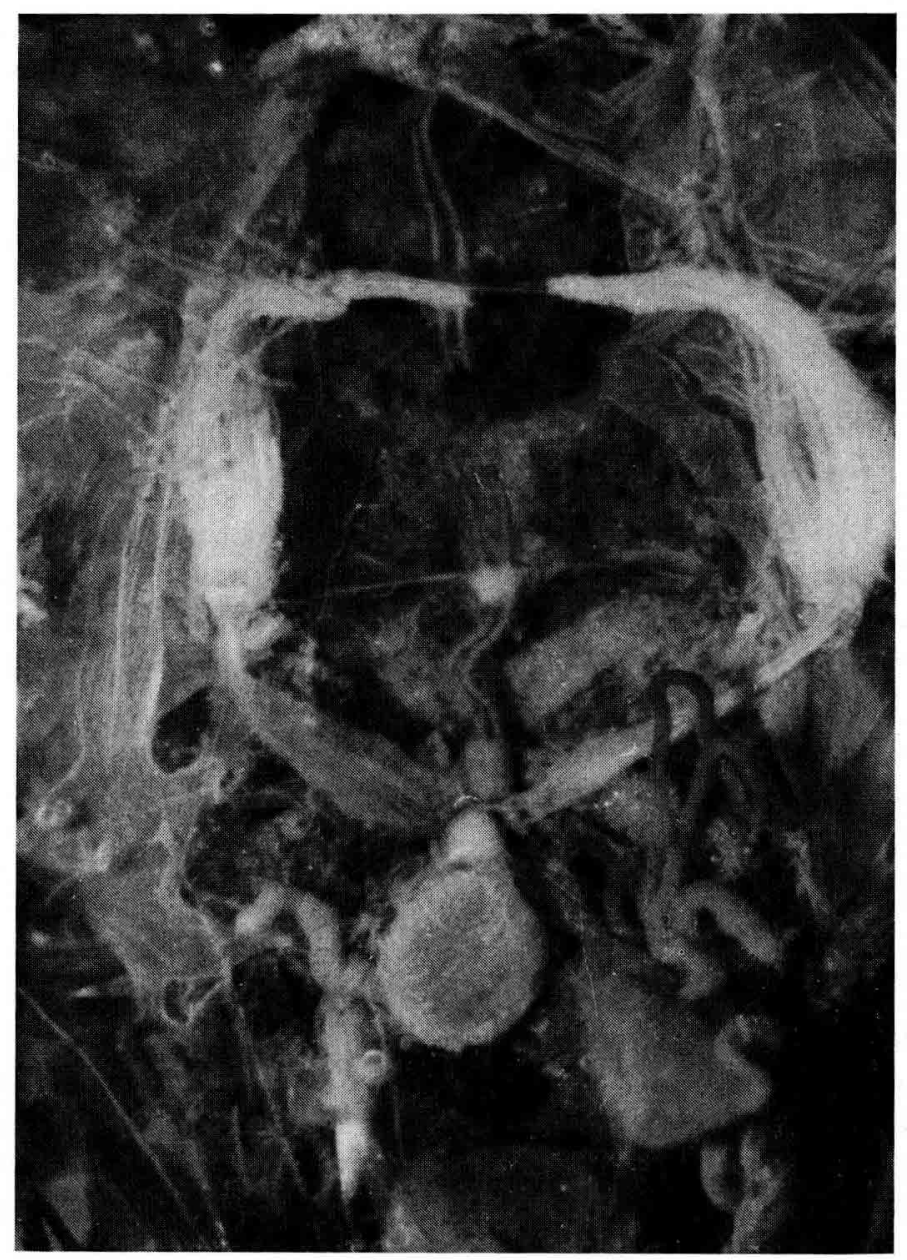

Aвв. 4. - Reproduktives System des Zwischentiers Nr. 9 aus Tabelle 2, Versuchsgruppe II

FIG. 4. -- Système reproducteur de l'individu intercaste $n^{\circ} 9$ du tableau 2, série d'expériences II.

\section{DISK USSION}

Das eindeutige Ergebnis dieser Versuche lautet : Auch im Arbeiterfuttersaft ist das königinnendeterminierende Prinzip wirksam. Bei der manuellen Aufzucht von Arbeitermaden mit Arbeiterfuttersaft kamen vorwiegend Arbeitsbienen zur Welt, doch entstanden auch ein Tier mit deutlichem Übergangscharakter und eine so gut wie kastentypische Königin. 
Königinnenmaden aus einem Pflegevolk, die im Alter von 3 Tagen nahe dem Ende ihrer Umstimmungsphase standen, reiften bei anschliessender manueller Fütterung mit Arbeiterfuttersaft zu königinnenhaften Tieren heran. Dagegen ergaben ihre gleichaltrigen und gleichschweren Schwestern nach der Umbettung in Arbeiterzellen mitten in das Brutnest eines weiselrichtigen Bienenvolkes - gefüttert vermutlich mit Arbeitermischfutter - äußerlich typische Arbeitsbienen, wenn auch mit zum Teil vermehrten Ovariolen und einer leicht verkürzten Entwicklungszeit. Auch die Divergenz in der Merkmalsausbildung der Tiere dieser beiden Versuchsgruppen weist auf das Vorhandensein eines königinnendeterminierenden Prinzips im Arbeiterfuttersaft hin. Es tritt offenbar durch die königliche Vorpflege der Larven besonders deutlich in Erscheinung.

Die von den Larven aufgenommene Futtermenge scheint für die Kastendetermination nicht belanglos zu sein. Die Tiere mit königlichen Merkmalen waren in meinen Versuchen in der Regel schwerer als Tiere vom Arbeiterinnentyp. Ganz allgemein scheinen sich bei Aufzuchtversuchen von Bienenmaden im Labor nur solche Tiere zu Königinnen zu entwickeln, die bis zu ihrer Verpuppung ein bestimmtes Mindestgewicht erreicht haben, wozu sie eine bestimmte Mindestmenge an Futter aufnehmen müssen. Anderenfalls entstehen Zwischenformen und Arbeiterinnen (Weaver 1957, Dietz und Lambremont 1971). Bei den Laboraufzuchten könnte die Futtersaftmenge insofern eine Rolle spielen, als mit ihr das determinierende Prinzip einer mengenmäßigen Regulation unterliegt.

Allerdings scheint diese Voraussetzung nicht in demselben Maß auch für die Königinnenaufzucht im Bienenvolk zu gelten. Im frisch von den Ammenbienen verabreichten Königinnenfutter ist das königinnenbestimmende Prinzip offenbar so reichlich vorhanden, daß auch bei einer erzwungenen Einstellung der Futterversorgung in der Verpuppungsphase zwar erheblich kleinere, aber doch immer noch königinnenhafte Tiere entstehen (s. Einleitung). Wahrscheinlich spielen hierbei aber auch Zeitpunkt und Zeitspanne der kastenspezifischen Futterversorgung mit.

Die Bedeutung der Futtersaftmenge für den Erfolg der Königinnenaufzucht im Labor berechtigt immerhin zu einem gewissen Zweifel am Vergleichswert der Aufzuchtversuche mit königlich vorgepflegten Maden im Labor und im Bienenvolk. In den Arbeiterzellen im Bienenvolk entstanden kleinere Tiere als bei der Laboraufzucht. Die räumliche Enge der Arbeiterzelle ist zwangsweise ein Begrenzungsfaktor des Größenwachstums der Larve. Sie könnte auch maßgebend für das weitgehende Ausbleiben der königlichen Entwicklung der in Arbeiterzellen umgebetteten Königinnenmaden gewesen sein.

Dagegen sprechen aber sehr eindringlich Versuche, die von Rheıs vor 
mehr als 40 Jahren (1933) angestellt hat. Er fütterte Königinnenlarven im Labor, also bei beliebiger Wachstumsmöglichkeit, mit Arbeitermischfutter. Während sich aus unter $20 \mathrm{mg}$ schweren Larven ausschließlich Arbeiterinnen entwickelten, zeigten die aus schwereren Larven gezogenen Tiere eine deutliche Vermehrung der Eiröhren und eine Vergrößerung der Samenblase. (Die unter $20 \mathrm{mg}$ schweren Königinnenlarven bekamen anfänglich Arbeiterfuttersaft, ehe sie mit Mischfutter weitergefüttert wurden, da sie sich sonst nicht entwickelt hätten.) Erst Tiere aus über $46 \mathrm{mg}$ schweren Königinnenlarven hatten auch eine gezahnte Mandibelkante. Dieses Madenalter liegt aber schon eindeutig jenseits des kritischen Umstimmungszeitpunktes der Kastenentstehung zwischen dem 3 . und 4. Larventag.

Von Rhein zog im Brutschrank auch Arbeiterlarven mit Mischfutter auf. Er erhielt aus über $45 \mathrm{mg}$ schweren Larven neben normalen Arbeitsbienen viele besonders schwere Tiere, von denen einige eine auffällige Vermehrung der Ovariolen und Vergrößerung der Samenblase aufwiesen. Selten schienen auch die Schlunddrüsen etwas zurückgebildet. Dagegen trat bei keiner dieser Puppen auch nur die geringste Abweichung in den äußeren Kastenmerkmalen vom Arbeiterinnentyp auf.

Wenn man in der verstärkten Ausbildung der reproduktiven Organe bereits einen echten königinnendeterminierenden Vorgang sehen will, muß man annehmen, daß auch im Arbeitermischfutter etwas von dem königinnendeterminierenden Prinzip enthalten ist. Schon wegen des Futtersaftanteils im Arbeitermischfutter läßt sich das nicht auschließen. Allerdings ist es sehr zweifelhaft, ob von RHEIN Futtersaft und Mischfutter auch immer genügend sauber voneinander getrennt geerntet hat. Er verließ sich bei der Futterausbeutung der Arbeiterzellen nicht auf altersdatierte Maden, sondern schätzte das Alter nach der Madengröße. Auch berichtet er, daß bei der Aufzucht im Brutschrank die Pollenkörner zu Boden sanken und einen Satz bildeten. Die Maden dürften deshalb hauptsächlich den Flüssiganteil des Futters aufgenommen haben, was bei der natürlichen Aufzucht im Bienenvolk sicher nicht in dieser Weise zutrifft.

Das determinierende Prinzip ist Bestandteil der Drüsenausscheidung der Ammenbiene. Nach unserem gegenwärtigen Wissen sind Hypopharyngealund Mandibulardrüse die hauptsächlichen, wenn nicht ausschließlichen Bildungszentren des Futtersaftes (Lit. bei Haydak 1968 und Örosi PaL 1968). HANser und Rembold (1964) vertreten, gestützt auf analytische, histologische und autoradiographische Untersuchungen dieser Drüsen bei weiselrichtigen und weisellosen Ammen die Auffassung, daß die Pharynxdrüse das Grundfutter produziere, welches mit dem Arbeiterfuttersaft identisch sei, während die für das Weiselfutter typischen Bestandteile aus der Mandibeldrüse kämen. Wenn letzteres in dieser Ausschließlichkeit zutrifft, kann man nach 
den Ergebnis.sen meintr künstlichen Aufzuchtversuche mit Arbeiterfuttersaft nur annehmen, daß auch im Arbeiterfuttersaft Mandibulardrüsensekret enthalten ist. Das meint auch Jung-Hoffmanv (1966), die beobachtete, daß Königinnenmaden und junge Arbeiterinnenlarven mit den gleichen Futtersaftkomponenten " weiß » und “ klar » gefüttert werden - nur in verschiedenem Verhältnis (s. Abschnitt I). Die Autorin stellte in chromatographischen und elektrophoretischen Untersuchungen fest, daß die weiße Komponente teils aus der Hypopharynx-, teils aus der Mandibeldrüse stammen müsse, während die klare Abscheidung aus der Pharynxdrüse und der Honigblase käme. In diesen Befund läßt sich die notwendige Schlußfolgerung aus meinen Versuchen, nämlich daß die königinnendeterminierende Komponente auch im Arbeiterinnenfuttersaft enthalten ist, zwanglos einfügen.

Das reproduktive System ist, wie die manuellen Aufzuchtversuche hinlänglich zeigen, gegenüber anderen Kastenmerkmalen besonders leicht zu beeinflussen. Ovar und Samenblase sind dabei nicht immer gleichsinnig betroffen. Warum - ist einstweilen nicht geklärt. Aber auch die unvollkommene Korrelation zwischen den inneren und äußeren und innerhalb der äußeren Kastenmerkmale gibt weitere Rätsel auf. Möglicherweise spielt bei der Ausbildung der reproduktiven Organe der reine Nährstoffaktor des Futters eine größere Rolle als bei den sekundären Kastenmerkmalen, oder das determinierende Prinzip greift zu bestimmten Zeitpunkten der Larvenentwicklung verschieden stark in die Ausbildung der einzelnen Kastenkriterien ein.

Auf die Frage nach dem Wesen des determinierenden Prinzips können die hier vorliegenden Untersuchungen keine Antwort geben. Es kann sich ebenso um einen einzelnen, noch unbekannten Wirkstoff handeln, den die Ammenbienen mit dem Futtersaft ausscheiden und der der Königinnenmade in größerer Menge zur Verfügung gestellt wird als der Arbeiterinnenmade, wie andererseits noch immer die Möglichkeit besteht, daß das Mengenverhältnis verschiedener bekannter essentieller Stoffe, einschließlich Grundnahrungsstoffe, von kastendeterminierender Bedeutung ist. Auch könnten beide Möglichkeiten gleichzeitig verwirklicht sein. Aus den vorliegenden Versuchen wird aber soviel deutlich, daß Königinnen- und Arbeiterinnenfuttersaft nicht zwei verschieden kastenspezifisch wirkende Substanzen sind, sondern daß sie sich höchstens im quantitativen Vorhandensein des gleichen Determinationsprinzips voneinander unterscheiden.

Eingegangen im November 1974 Reçu pour publication en novembre 1974

\section{RESUME}

Dans la ruche les larves d'ouvrières reçoivent comme nourriture de la gelée d'ouvrière durant les 3 à 4 premiers jours, puis un mélange de pollen, de miel et de secrétion des glandes. Jusqu'au changement de nourriture, les larves d'ouvrières croissent sur de la gelée royale 
comme des reines typiques, que ce soit en élevage naturel dans la ruche ou en élevage artificiel en laboratoire. Tout en ne connaissant pas à l'heure actuelle le principe qui détermine les castes et qui est présent dans la nourriture larvaire royale, on peut se demander si ce principe ne serait pas également actif dans la gelée d'ouvrière. SHuEL et Dixon (1968) ont montré que des larves d'ouvrière nourries de gelée d'ouvrière peuvent atteindre le stade de nymphe si l'on y ajoute du sucre; la poursuite de cette expérience s'imposait afin d'engendrer des adultes et d'en étudier la caste.

Dans une colonie pourvue d'une reine, j'ai prélevé de la gelée d'ouvrière dans des cellules de larves âgées de 2 jours $1 / 4$ au plus et l'ai enrichie d'une solution à $10 \%$ de sucre inverti, tout en maintenant la teneur naturelle en eau à environ $70 \%$. Avec cette gelée j'ai élevé en étuve des larves d'ouvrières âgées de 2 jours à 2 jours $1 / 4$, et dans un cas de 2 jours $3 / 4$ à 3 jours, selon la méthode connue. Il en est mort $15,2 \%$ au stade de larve enroulée, $59,1 \%$ à celui de prépupe et $7,6 \%$ à celui de pupe. J'ai obtenu 12 (soit 18,1\%) d'insectes adultes ou presque adultes, dont j'ai examiné le poids, la durée de développement, le nombre d'ovarioles, la configuration des mandibules et des pattes postérieures ainsi que l'appareil venimeux. Dix d'entre eux étaient des ouvrières, ou tout au moins en étaient très proches; seules les spermathèques étaient un peu plus grosses et parfois une mandibule ou un aiguillon différait légèrement de celle ou celui des ouvrières. Toutefois un insecte était un intercaste typique selon les critères étudiés, et un autre une reine complète (tableau 2, série d'expériences II). Il ressort de cela que le principe déterminant des castes est également présent dans la gelée d'ouvrière - quoique sous une forme atténuée, vraisemblablement.

Dans une autre série d'expériences en laboratoire, j’ai nourri des larves de reine âgées d'environ 3 jours avec de la gelée d'ouvrière sucrée. La mortalité, prédominante au stade de prépupe, s'est élevée à $66,7 \%$ et 7 insectes $(33,3 \%$ ) sont parvenus jusqu'à la mue imaginal e Tous les 7 avaient un type reine très prononcé (tableau 2, série d'expériences III).

A titre de comparaison j'ai placé des larves de reine de même âge dans des cellules d'ouvrières d'un rayon de couvain non operculé, dont j'avais ôté auparavant des larves d'ouvrières, et ai introduit le rayon dans le nid à couvain d'une colonie pourvue d'une reine. Les larves de reine transférées ont donné naissance à des ouvrières en apparence parfaites, qui possédaient néanmoins un nombre d'ovarioles en partie plus élevé tout en ayant une spermathèque d'ouvrière remarquablement inchangée. Il semble qu'à plusieurs reprises la durée de développement aie été quelque peu raccourcie (tableau 2, série d'expériences IV).

La prédétermination royale des larves, laquelle dans les séries d'expérience III et IV - donc en élevage au laboratoire (avec de la gelée d'ouvrière) et en élevage dans la colonie (probablement avec de la nourriture mixte d'ouvrière) - doit s'être produite de façon analogue, sembla d'abord rendre réellement actif le principe déterminant royal dans la gelée d'ouvrière.

La capacité limitée de croissance que présentent les larves d'expérience dans les cellules d'ouvrières constitue le point critique de la série d'expériences IV. Si l'on prend en considération le fait que von RHein (1933) a obtenu, en élevant en laboratoire des larves de reine sur de la nourriture mixte d'ouvrière, des ouvrières typiques du point de vue externe, bien que partiellement “ géantes », et qui ne présentaient qu'un plus grand développement des ovaires et de la spermathèque, il faut bien admettre que l'aspect d'ouvrière de mes insectes, élevés in vivo dans des cellules d'ouvrières à partir des larves de reine, ne peut pas être imputé à la seule étroitesse du réceptacle d'élevage mais également au type de nourriture administrée par les nourrices.

Du reste les expériences d'élevage en laboratoire avec de la gelée d'ouvrière montre que la plus grande taille que les larves peuvent atteindre, ainsi que la quantité de gelée ingérée, sont de quelque importance. Des prépupes de petite taille se sont toujours développées en ouvrières, 
lorsqu'elles ont survécu. Au moins en laboratoire le principe de détermination des castes semble être soumis avec la quantité de gelée consommée par les larves à une régulation quantitative.

\section{LITERATURVERZEICHNIS}

O'Brien D. M. u. Shuel R. W., 1972. - In fluence of diet upon the development of prothoracic glande and oenocytes in female honeybee larvae. J. apic. Res. 11 (1) 13-21.

BuchNer R., 1953. - Beein flussung der Größe der Arbeitsbiene durch Raum- und Nahrungsmangel während der Larvenzeit. Wilh. Roux' Arch. Entwicklungsmech. 146, 544-579.

Butenandt A. u. Rembold H., 1957. - Über den Weiselzellenfuttersaft der Honigbiene I. Isolierung, Konstitutionsermittlung und Vorkommen der 10-hydroxy- $\triangle^{2}$-decensäuere. Hoppe Seyl. Z. physiol. Chemie 308, 284-289.

Butenandt A. u. Rembolo H., 1958. - Über den Weiselzellenfuttersaft der Honigbiene. II. Isolierung von 2-amino-4-hydroxy-6-[1,2-dihydroxy-propyl-]-pteridin. Hoppe Seyl. Z. physiol. Chemie 311, 79-83.

Canetty S. J., Shuel R. W. u. Dixon S. E., 1964. - Studies in the mode of action of royal jelly in honeybee development. IV. Development within the brain and retrocerebral complex of female honeybee larvae. Can. J. Zool, 42, 229-233.

Czoppelt Ch. u. Rembold H., 1967, - Ein fluß des weiblichen Dimorphismus der Honigbiene auf entwicklungsabhängige Enzymaktivitäten des Kohlenhydratstoffwechsels. Hoppe Seyl. Z. physiol. Chem. 348, S. 1229.

Czoppet Ch. u. Rembold H., 1970. - Vergleichende Analyse des Kohlenhydratstoffwechsels bei den Kasten der Honigbiene, Apis mellifera. J. Ins. Physiol. 16, 1249-1264.

DieTz A. u. HAYDAK M. H., 1971. - Caste determination in honey bees. I. The significance of moisture in larval food. J. Exp. Zool. 177 (3) 353-358.

Dietz A. u. Lambremont E. M., 1970. - Caste determination in honey bees. II. Food consumption of individual honey bee larvae, determined with ${ }^{32} \mathrm{P}$-labeled royal jelly. Ann. Ent. Soc. Amer. 63 (5) 1342-1345.

Dixon S. E. u. Shuel R. W., 1963. - Studies in the mode of action of royal jelly in honeybee development. III. The effect of experimental variation in the diet and growth and metabolism of honeybee larvae. Canad. J. Zool. 41, 733-739.

Dönhoff E., 1859. - Über die künstliche Erziehung von Zwergköniginnen. Eichst. Bztg. 15, 8-9.

Gontanski H., 1953. - Zur Brutbiologie der Honigbiene. Z. Bienenf. 2, 1-4.

Gontarski H., 1954. - Untersuchungen über die Verwertung von Pollen und Hefe zur Brutpflege der Honigbiene. Z. Bienenf. 2 (6) 161-180.

Hanser Gisela u. Rembold H., 1960. -- Über den Weiselzellenfuttersaft. IV. Jahreszeitliche Veränderungen im Biopteringehalt des Arbeiterinnenfuttersaftes. Hoppe Seyl. Z. physiol. Chem. 319, 200-205.

Hanser Gisela u. Rembold H., 1964. - Analytische und histologische Untersuchungen der Kopf- und Thoraxdrüsen bei der Honigbiene Apis mellifica. Z. Naturforsch. 19 (10) 938-943.

HAYDAK M. H., 1935. - Brood rearing by honeybee confined to a pure carbohydrate diet. J. econ. Ent. 28, 657-660.

HAYDAK M. H., 1943. - Larval food and development of castes in the honeybee. J. econ. Ent. 36, 778-792.

HaYDAK M. H., 1968. - Nutrition des larves d'abeilles. In Chauvin : Traité de biologie de l'abeille 1, 302-333, Masson et Cie, Paris.

HaYdaK M. H. u. Vivino A. E., 1950. - Changes in the thiamine, riboflavin, niacin and pantothenic acid content in the food of female honey-bee during growth with a note on vitamin $K$ activity of royal jelly and bee bread. Ann. Ent. Soc. Amer. 43, 361-367.

Himmer A., 1927. - Fortschritte auf dem Gebiet der Bienenkunde und der Bienenzucht. Erl. Jahrb. 5, 70-71. 
JAY S. C., 1959. - Factors affecting the laboratory rearing of honey bee larvae (Apis mellifera L.). Univ. Toronto. M.S.A. Thesis.

JAY S. C., 1961. - Laboratory studies on the rearing, growth and development of the honey bee (Apis mellifera L.). Univ. London Ph. D. Thesis.

JAY S. C., 1964. - Starvation studies of larval honey bees. Canad. J. Zool. 42, 455-462.

Johansson R. S. K. u. Johansson M. P., 1958. - Royal jelly II. Bee World 39, 254-264, 277-286.

Jung-Hoffmann, Irmgard, 1966. - Die Determination von Königin und Arbeiterin der Honigbiene. Z. Bienenf. 8 (9) 296-322.

Kuwabara. M., 1947. - Über die Regulation im weisellosen Volke der Honigbiene (Apis mellifica), besonders die Bestimmung des neuen Weisels. J. Fac. Sci. Hokkaido Univ. VI Zool. 9, 359-381.

Lingens F. u. Rembold H., 1959. - Über den Weiselzellenfuttersaft der Honigbiene. III. Vitamingehalt von Königinnen- und Arbeiterinnenfuttersaft. Hoppe Seyl. Z. physiol. Chemie 314, 141-146.

Liv T. P. u. Dixon S. E., 1965. - Studies in the mode of action of royal jelly in honeybee development. VI. Haemolymph protein changes during caste development. Can. J. Zool. 43, 873-879.

Lue P. F. u. Dixon S. E., 1967. - Studies in the mode of action of royal jelly in honeybee development. VII. The free amino acids in the haemolymph of developing larvae. Can. J. Zool. 45, 205-214.

Melampy R. M. u. Willis E. R., 1939. - Respiratory metabolism during larval and pupal development of the female honeybee. Physiol. Zool. 12, 302-311.

Melampy R. M., Willis E. R. u. McCregor S. E., 1940. - Biochemical aspects of the differentiation of the female honeybee (Apis mellifica L.). Physiol. Zool. 13, 283-293.

Mickey G. H. u. Melampy R. M., 1941. - Cytological Studies on fat cells in the larval honeybee (Apis mellifera L.). Anat. Rec. 81 Suppl. 53; bei ShuEl u. Dixon, 1960. Ins soc. VII (3) S. 267.

Örösi Pál Z., 1968. - La physiologie des glandes nourricières. In Chauvin : Traité de biologie de l'abeille 1, 263-290, Masson et Cie, Paris.

Osanai M. u. Rembold H., 1968. - Entwicklungsabhängige mitochondriale Enzymaktivitäten bei den Kasten der Honigbiene. Biochem. Biophys. Acta 162, 22-31.

Rembold H., 1964. - Die Kastenentstehung bei der Honigbiene Apis mellifica L. Naturwiss. 51 (3) $49-54$.

Rembold H., 1969. - Biochemie der Kastenentstehung bei der Honigbiene. Proc. IV. Congr. IUSSI Bern. 239-246.

Rembold H., 1973. - Biochemie der Kastenbildung bei der Honigbiene. Naturw. Rundschau 26 (3) 95-102.

Rembold H. u. Buschmann L., 1963. - Struktur und Synthese des Neopterins. Chem. Ber. 96, 1406-1410.

Rembold H. u. Hanser Gisela, 1964. - Über den Weiselzellenfuttersaft der Honigbiene. VIII. Nachweis des determinierenden Prinzips im Futtersaft der Königinnenlarven. Hoppe Seyl. Z. physiol. Chem. 339, 251-254.

Rembold H., Czoppelt Ch. u. Rao P. J., 1974. - Effect of juvenile hormone treatment on caste differentiation in the honeybee, Apis mellifera. J. Insect Physiol, 20, 1193-1202.

Rheis W. v., 1933. - Über die Entstehung des weiblichen Dimorphismus im Bienenstaate. Wilh. Roux' Arch. Entw. Mech. Org. 129 (4) 601-665.

Shuel R. W. u. Dixon S. E., 1959. - Studies in the mode of action of royal jelly in honeybee development. II. Respiration of newly emerged larvae on various substrates. Can. J. Zool. 37, 803-813.

Shuel R. W. u. Dixon S. E., 1960. - The early establishment of dimorphism in the female honeybee, Apis mellifera L. Ins. soc. 7 (3) 265-282.

Shuel R. W. u. Drxon S. E., 1968. - Respiration in developing honeybee larvae. J. apic. Res. 7 (3) 11-19. 
Shuel R. W. u. Dixon S. E., 1968. - The importance of sugar for the pupation of the worker honeybee. J. apic. Res. 7 (3) 109-112.

Sмiтн M. V., 1959. - The production of royal jelly. Bee World 40 (10) 250-254.

Sмiтн M. V., 1959. - Queen differentiation and the biological testing of royal jelly. Mem. Cornell agric. Exp. Sta. No 356.

STABE H. A., 1930. - The rate of growth of worker, drone and queen larvae of the honeybee, Apis mellifera L. J. econ. Ent. 23, 447-453.

Straus J., 1911. - Die chemische Zusammensetzung der Arbeitsbienen und Drohnen während ihrer verschiedenen Entwicklungsstadien. Z. Biol. 56, 347-397.

Townsend G. F. u. Shuel R. W., 1962. - Some recent advances in apicultural research. Ann. Rev. Ent. 7, 481-500.

Tripathi R. K. u. Dixon S. E., 1968. - Haemolymph esterases in the female larvale honeybee, Apis mellifera L., during caste development. Can. J. Zool. 46, 1013-1017.

Tripathi R. K. u. Dixon S. E., 1969. - Changes in some haemolymph dehydrogenase isozymes of the female honeybee Apis mellifera L., during caste development. Can. J. Zool. 47, 763-770.

Wang Der-J, 1965. - Growth rates of young queen and worker honeybee larvae. J. apic. Res. 4 (1) 3-5.

W ANG Der-J u. Shuel R. W., 1965. - Studies in the mode of action of royal jelly in honeybee development. V. The influence of diet on ovary development. J. apic. Res. 4 (3) 149-160.

Weaver N., 1956. - Ovarian development of worker honeybees. J. econ. Ent. 49, 854-857.

Weaver N., 1957. - Effects of larval age on dimorphic differentiation in the female honeybee. Ann. Ent. Soc. Amer. 50 (3) 283-294.

WeAver N., 1958. - Rearing honeybee larvae in the laboratory. X. Int. Congr. Ent. 1956, 4, 1031-1036.

Weaver N., 1962. - Control of dimorphism in the female honeybee. Science, N.Y. 138, S. 995.

Weaver N., 1974a. - Control of dimorphism in the female honeybee. 2. Methods of rearing larvae in the laboratory and of preserving royal jelly. J. apic. Res. 13 (1) 3-14.

Weaver N., 1974b. - Control of dimorphism in the femal honeybee. 3. The balance of nutrients. J. apic. Res. $13(2)$ 93-101.

WEISs K., 1967. - Zur vergleichenden Gewichtsbestimmung von Bienenköniginnen. Z. Bienenf. 9 (1) $1-21$.

WEIss K., 1971. - Über Ausbildung und Leistung von Königinnen aus Eiern und jungen Arbeitermaden. Apidologie 2 (1) 2-47.

Weiss K., 1974. - Zur Frage des Königinnengewichtes in Abhängigkeit von Umlarvalter und Larvenversorgung. Apidologie 5 (2) 127-147.

Whitcomв W. u. WiLson H. F., 1927. - A suggested explanation of why bees appear to use pollen substitudes for brood rearing. Amer. Honey Producer 1 (3) 36-38.

Wirtz P., 1973. - Differentiation in the honeybee larva. Meded. Landbouwhogeschool Wageningen, Nederland 73-5, 1-155.

Wirtz P. u. BeEtsma J., 1972. - Induction of caste differentiation in the honeybee (Apis mellifera) by juvenile hormone. Ent. exp. u. appl. 15, 517-520. 